\title{
Water vapor masers in stars departing from the AGB
}

\author{
D. Engels* \\ Hamburger Sternwarte, Gojenbergsweg 112, 21029 Hamburg, Germany
}

Received 17 December 2001 / Accepted 25 March 2002

\begin{abstract}
The $22 \mathrm{GHz}$ water vapor masers of four "non-variable" OH/IR stars considered to be young ProtoPlanetary Nebulae (PPNs) were monitored for more than 10 years. In two of them, OH 15.7+0.8 and OH 17.7-2.0 having Mira-like line profiles, the maser flux decreased and the maser finally disappeared. The decrease of the maser brightness is spectacular for $\mathrm{OH}$ 17.7-2.0 in particular. The maser had a flux density of several hundred Jansky in the eighties and is now absent since 1990. The masers of $\mathrm{OH} 15.7+0.8$ and $\mathrm{OH} 17.7-2.0$ probably emerged in the "dying AGB wind" during the mass loss reduction process accompanying the departure from the AGB. The other two, OH 12.8-0.9 and OH 37.1-0.8, have double-peaked profiles with a considerably larger velocity spread than the $\mathrm{OH}$ masers in these sources. The velocities are incompatible with the spherical symmetric mass loss process on the AGB and give evidence for the presence of probably bipolar outflows with a projected velocity of $v_{\mathrm{e}} \approx 28 \mathrm{~km} \mathrm{~s}^{-1}$ during the very early PPN phase. The gross structure of their profiles was rather stable during the monitor period while individual maser lines appeared and disappeared. An increase of the velocity range was found in both sources implying that the outflow is accelerating. Analysis of the profile variations of all four objects suggests a lifetime of individual maser components of 1-3 years. There is no evidence for ordered motion traced by the masers. The $\mathrm{H}_{2} \mathrm{O}$ maser properties of all these objects are strongly different from those of ordinary $\mathrm{OH} / \mathrm{IR}$ stars, allowing to find new young PPNs among optically still hidden objects by looking for irregular maser profiles. In contrast to current definitions in the literature "non-variable" OH/IR stars are proposed to be classified as (young) PPNs. It is argued that beyond the end of the AGB, maser properties cannot be used to infer the evolutionary stage of the objects in transition to Planetary Nebulae.
\end{abstract}

Key words. stars: AGB and Post-AGB - masers - circumstellar matter

\section{Introduction}

At the end of the stellar evolution on the Asymptotic Giant Branch (AGB) stars loose copious amounts of mass, which build up a circumstellar dust and gas shell hiding the star from optical view almost completely. Stars departing from the AGB and evolving towards the Planetary Nebula (PN) phase are therefore difficult to observe optically, and the details of the transition process from the AGB to the PN phase, the stage when the nebula is optically prominent again, remains to be uncovered. Only with the IRAS All-Sky Survey a sizeable number of far-infrared emitters were discovered, which are now considered as transition objects, and allow observational studies of the transition process. These transition objects are referred to as Post-AGB stars or Proto-Planetary Nebulae (PPNs). They are identified by searching for stars in optical catalogues with appropriate IRAS colors or by searching for optical counterparts to extremely cool IRAS sources (Kwok 1993). This search technique is sensitive to stars, in which the AGB mass loss has stopped and the remnant envelope has been diluted so far that optical emission from

\footnotetext{
* e-mail: dengels@hs.uni-hamburg.de
}

the central star is visible again. These stars typically have effective temperatures $\gtrsim 5000 \mathrm{~K}$ and spectral types earlier than K. Stars with $T_{\text {eff }} \approx 2000-5000 \mathrm{~K}$ in an evolutionary stage between the end of the thermal pulse phase on the AGB (from the observational point of view defined here as the end of the large amplitude pulsations and of the corresponding high mass loss rates) and the stage, in which they reappear optically, will not be found. There is however a fraction of the classical OH/IR stars, which is considered to contain candidate objects (named "young PPNs" hereafter) for this evolutionary stage. These are the "non-variable" $\mathrm{OH} / \mathrm{IR}$ stars, which do not vary at all or aperiodically with small amplitude (Habing et al. 1987; Bedijn 1987).

Besides of having high mass loss rates, stars in the thermal pulse phase of AGB evolution generally are large amplitude long-period variables and, in the case they are oxygen rich, often strong sources of $\mathrm{OH}, \mathrm{H}_{2} \mathrm{O}$, and $\mathrm{SiO}$ maser emission. During the transition process somehow the variability has to cease and the stars get lost of their masers, which are located in different shells with increasing distance from the star. The innermost is the $\mathrm{SiO}$ maser shell, followed by $\mathrm{H}_{2} \mathrm{O}$, and the outermost is occupied by 
Table 1. Coordinates and circumstellar shell velocity parameters for the observed "young PPNs". $v_{\mathrm{c}}$ is the radial velocity of the star and $v_{\mathrm{e}}$ is the expansion velocity of the old AGB wind. $v_{\mathrm{c}}$ and $v_{\mathrm{e}}$ were derived from the $1612 \mathrm{MHz} \mathrm{OH}$ maser profile. $v_{\mathrm{c}}^{\mathrm{H}_{2} \mathrm{O}}$ give the center of the $\mathrm{H}_{2} \mathrm{O}$ maser velocity interval and $v_{\mathrm{e}}^{\mathrm{H}_{2} \mathrm{O}}$ its outflow velocity.

\begin{tabular}{|c|c|c|c|c|c|c|c|c|}
\hline Object & \multicolumn{2}{|c|}{ Coordinates (1950) } & Ref. & $v_{\mathrm{c}}$ & $v_{\mathrm{e}}$ & Ref. & $v_{\mathrm{c}}^{\mathrm{H}_{2} \mathrm{O}}$ & $v_{\mathrm{e}}^{\mathrm{H}_{2} \mathrm{O}}$ \\
\hline$O H \quad 157+08$ & $1812 \quad 23$ & 1456.11 & 1 & 06 & 160 & 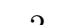 & 02 & $655+2+3$ \\
\hline OH $12.8-0.9$ & 181353.9 & -181607 & 3 & -55.5 & 14 & 4,5 & -58 & 28 \\
\hline $\mathrm{OH} 17.7-2.0$ & 182739.8 & -143104 & 6 & 61.4 & 14.1 & 2,7 & 58.9 & 8.6 \\
\hline $\mathrm{OH} 37.1-0.8$ & 185936.2 & +031553 & 2,8 & 88.5 & 14.9 & 2 & 88.5 & 28.5 \\
\hline
\end{tabular}

References: 1) Bowers et al. (1980), 2) Herman et al. (1985), 3) Bowers \& de Jong (1983), 4) Baud et al. (1979), 5) Dickinson \& Turner (1991), 6) Bowers et al. (1981), 7) Heske et al. (1990), 8) Gómez et al. (1994).

the $\mathrm{OH}$ masers. It is generally assumed that the AGB evolution terminates with the exhaustion of nuclear fuel, which is connected to a decrease in the degree of variability, and to a decrease of the mass loss rates. The circumstellar shell is then not further replenished with material and remains a few thousand years as remnant shell, while it is swept up by a new fast wind which is fully developed when the star is already a young planetary nebula. Thus, when the high AGB mass loss rates stop the masers should disappear one after another, beginning with $\mathrm{SiO}$, followed by $\mathrm{H}_{2} \mathrm{O}$ and at the end several thousand years after the beginning of the drop in mass loss rates by the $\mathrm{OH} 1612 \mathrm{MHz}$ masers.

The restrengthening of the main line $\mathrm{OH}$ masers at 1665 and $1667 \mathrm{MHz}$ among PPNs compared to the $1612 \mathrm{MHz}$ satellite line $\mathrm{OH}$ masers (Lewis 1989) is observational evidence indicating that the transition process is more complex, probably because of two reasons. First, the AGB wind might not stop abruptly but decrease steadily over a period of time, in which the properties in the inner circumstellar shell may resemble those of AGB stars of relatively low mass loss rates (SR variables and Miras). Second, the development of the bipolar structures commonly seen in PPNs and PNs may start already when the star is still on the AGB and may influence the appearance of the masers. These bipolar structures are connected with a new wind, faster than the old AGB wind, which might be able to sustain maser emission as well, allowing the presence of maser emission from both winds at a time.

We will present here $\mathrm{H}_{2} \mathrm{O}$ maser emission of several young PPNs, which we interpret as evidence for the happening of processes as described before. The data consist of multi-epoch radio observations of $\mathrm{OH} 17.7-2.0$ and $\mathrm{OH} 15.7+0.8$, in which the disappearance of the $\mathrm{H}_{2} \mathrm{O}$ masers imply a current decrease of the mass loss rate on short timescales, and of $\mathrm{OH} 12.8-0.9$ and $\mathrm{OH} 37.1-0.8$, in which the $\mathrm{H}_{2} \mathrm{O}$ masers probably trace the emergence of the new Post-AGB wind. The stars are part of a larger monitoring program of $\mathrm{H}_{2} \mathrm{O}$ masers in $\mathrm{OH} / \mathrm{IR}$ stars and are presented here separately because of their peculiarity. Basic data for the four objects are tabulated in Table 1. An early report of the program was given by Engels (1997).
The outline of the paper is as follows. In Sects. 2 and 3 we describe the observations, the data reduction and method applied to analyze the line profiles. In Sect. 4 we present the results of the profile analysis. In Sect. 5 we discuss the origin of the $\mathrm{H}_{2} \mathrm{O}$ maser emission in these objects and conclude the paper in Sect. 6 with a discussion on search strategies of PPNs, on the delimitation between AGB stars and PPNs, and on maser properties during the transition process.

\section{Water vapor maser observations at $22 \mathrm{GHz}$}

The observations of the $\mathrm{H}_{2} \mathrm{O}$ maser line at $22235.08 \mathrm{MHz}$ were made during various epochs between 1987 and 1999 using the 100 m-radiotelescope in Effelsberg. A log of the observations is included in Tables 2 to 6 , which list the results of the line profile analyses from Sect. 4 . The instrumental equipment and the observing technique is described in Engels \& Lewis (1996). Due to varying weather conditions and depending on elevation the system temperatures were in the range $80-200 \mathrm{~K}$, allowing the detection of 0.1 to $1 \mathrm{Jy}$ maser lines with integration times of $10 \mathrm{~min}$ $(\mathrm{ON}+\mathrm{OFF})$. A bandwidth of $6.25 \mathrm{MHz}$ was used giving spectra with a velocity resolution of $0.16 \mathrm{~km} \mathrm{~s}^{-1}$ after Hanning smoothing and a velocity coverage of $80 \mathrm{~km} \mathrm{~s}^{-1}$. The pointing accuracy was regularly checked with strong continuum sources.

Interferometric positions are available for all objects (Table 1 ), so that the objects were always well centered $\left(<10^{\prime \prime}\right)$ within the beam, which has a FWHM of $\approx 40^{\prime \prime}$. Note that the declination of the interferometric $\mathrm{OH}$ maser position of $\mathrm{OH} 15.7+0.8$ given by Bowers et al. (1980) has an error of $11^{\prime \prime}$. The $\mathrm{OH}$ maser position determined later by Fix \& Mutel (1984) is inconsistent with Bowers' coordinates. By offsetting the telescope we found, that the position of the $\mathrm{H}_{2} \mathrm{O}$ maser is consistent with the Bowers et al. coordinates and that their errors are $<10^{\prime \prime}$. Also in this case the telescope was always pointed correctly. Recently Szymczak \& Richards (2001) confirmed Bowers' coordinates.

Data reduction was made using the CLASS spectral line reduction software. The spectra were calibrated relative to NGC 7027 and derived flux values are estimated 
to be accurate within $20 \%$. A representative selection of spectra taken during the monitoring program is given in Figs. 1-4.

To study the overall longterm variations of the masers integrated fluxes $S_{\text {I }}$ were determined (cf. Tables 2 to 6$)$. They were taken over the full velocity range $v_{\mathrm{c}}^{\mathrm{H}_{2} \mathrm{O}} \pm\left(v_{\mathrm{e}}^{\mathrm{H}_{2} \mathrm{O}}+1 \mathrm{kms}^{-1}\right)$ as given in Table 1, in which $\mathrm{H}_{2} \mathrm{O}$ maser emission has been detected. The baselines for all spectra of a particular source were determined using fixed velocity intervals outside the maser velocity range, to avoid biases due to baseline determination. This is very critical for $\mathrm{OH} 12.8-0.9$ and $\mathrm{OH} 37.1-0.8$ with their large velocity ranges and their weak redshifted features (Figs. 3, 4). An asystematic baseline determination introduces easily larger integrated flux variations between two spectra, than the presence or absence of weak features. Although carefully determined, the integrated fluxes may have errors up to $50 \%$.

\section{Line profile analysis methods}

A detailed analysis of the variations of the $\mathrm{H}_{2} \mathrm{O}$ maser profiles was made to test for the presence of subtle velocity shifts due to ordered motions. Such shifts might be present, if the masers are located in accelerated outflows, as it has been observed for the $\mathrm{OH}$ masers of the PPN HD 101584 (te Lintel Hekkert et al. 1992). They are difficult to detect however because of blending problems and the limited lifetime of the maser components.

$\mathrm{H}_{2} \mathrm{O}$ masers usually are made up by many individual maser lines, forming blended features. To determine velocities and flux densities of individual maser lines, multiplecomponent Gauss fits were applied to adjacent groups of lines. The Gauss fitting routine of the CLASS package was used, which allows to fit up to five components simultaneously. Each spectrum was first fitted without knowledge from spectra taken before or afterwards. In a second iteration it was verified that no inconsistencies occurred. These fits proved to be powerful to distinguish between different lines as long as the signal-to-noise ratio of the lines is sufficient. In cases, where lines in blended features have similar intensity, the separation is difficult, unless the half-width of the lines is fixed. When Gauss fitting failed for weak maser lines, velocities and flux densities were determined interactively with the cursor. If lines are not fitted well with a Gaussian, the peak fluxes are underestimated by 10-20\% and corrections were applied for this.

To derive velocities in the $\mathrm{H}_{2} \mathrm{O}$ maser region relative to the star we use the $\mathrm{OH}$ maser observations (1612 and $1667 \mathrm{MHz}$ ) to determine the radial velocity of the star and the expansion velocity of the circumstellar shell originating from the AGB wind. All sources discussed here have classical double peaked $1612 \mathrm{MHz} \mathrm{OH}$ spectra, so that we can assume the standard model of a radially symmetric $\mathrm{OH}$ maser shell to be valid. The half width of the $\mathrm{OH}$ maser velocity range gives then the expansion velocity $v_{\mathrm{e}}$ of the shell and the midpoint $v_{\mathrm{c}}$ the radial velocity of the star. For OH 17.7-2.0 the profile of a CO-line detected by
Heske et al. (1990) can be used in addition. Their values are given in Table 1.

The determination of outflow velocities in the $\mathrm{H}_{2} \mathrm{O}$ maser region is not as straightforward as for $\mathrm{OH}$. While the $\mathrm{OH}$ shell has a constant outflow velocity, the $\mathrm{H}_{2} \mathrm{O}$ maser shell might be located in accelerating parts of the shell. Then, provided the $\mathrm{H}_{2} \mathrm{O}$ velocity range is centered on $v_{\mathrm{c}}$, its half width gives the upper limit of a range of outflow velocities. To determine the width of the $\mathrm{H}_{2} \mathrm{O}$ velocity range is not easy, as unlike for $\mathrm{OH}$ masers, the individual $\mathrm{H}_{2} \mathrm{O}$ lines do not necessarily appear close to the edges of the range. However, by monitoring the maser the probability increases to detect a line at the edge some time. Determining the velocity difference between the most redshifted or most blueshifted $\mathrm{H}_{2} \mathrm{O}$ maser line observed at any epoch and the radial velocity $v_{\mathrm{c}}$ of the star will give then the best estimate for the maximum expansion velocity $v_{\mathrm{e}}^{\mathrm{H}_{2} \mathrm{O}}$ of the $\mathrm{H}_{2} \mathrm{O}$ maser shell. The best estimates for $v_{\mathrm{c}}^{\mathrm{H}_{2} \mathrm{O}}$ and $v_{\mathrm{e}}^{\mathrm{H}_{2} \mathrm{O}}$ as derived in Sect. 4 are compiled in Table 1.

\section{Results of the profile analysis}

The results of the line fitting are given individually for each source in Tables 2 to 6 . These tables give the observing date in Gregorian and Julian (JD) format, the rmsnoise of the spectra, the integrated flux $S_{\mathrm{I}}$, and velocities $v_{\mathrm{p}}$ and flux densities $S_{\mathrm{p}}$ of the peaks of individual components identified. For a given epoch, lines were included in the tables, if either they can be identified unambiguously in the correspondent spectrum or are considered to be real by comparison with other spectra. Less secure lines are denoted by a colon. The lines were assigned to different components using the similarity of velocities as criterium. Ideally each of the maser components would correspond to one individual maser cloud giving rise to one narrow emission line at a particular velocity. In practice however, blending and limited lifetime make the components often superpositions of lines coming from physically different maser clouds. The definition of the components and the assignment of lines to them is therefore subjective to some degree. Interferometric monitoring on time scales of months would be required to improve on it. The accuracy of the velocities also depends on the degree of blending, so that velocity shifts of $\$ 0.3 \mathrm{~km} \mathrm{~s}^{-1}$ of individual components between two observing epochs are not necessarily real shifts.

\section{1. $\mathrm{OH} 15.7+0.8$}

The $\mathrm{H}_{2} \mathrm{O}$ maser of $\mathrm{OH} 15.7+0.8$ was discovered by Engels et al. (1986), displaying during three epochs in $1984 / 85$ several lines in the velocity range from -4.5 to $+6.3 \mathrm{~km} \mathrm{~s}^{-1}$ with peak flux densities of $4-5 \mathrm{Jy}$. A consistent spectrum was measured by Benson \& Little-Marenin (1996) in 1985 January. In 1986 December they obtained a second spectrum showing a complex profile with several lines in the velocity range -7 to $+6 \mathrm{~km} \mathrm{~s}^{-1}$. This spectrum 

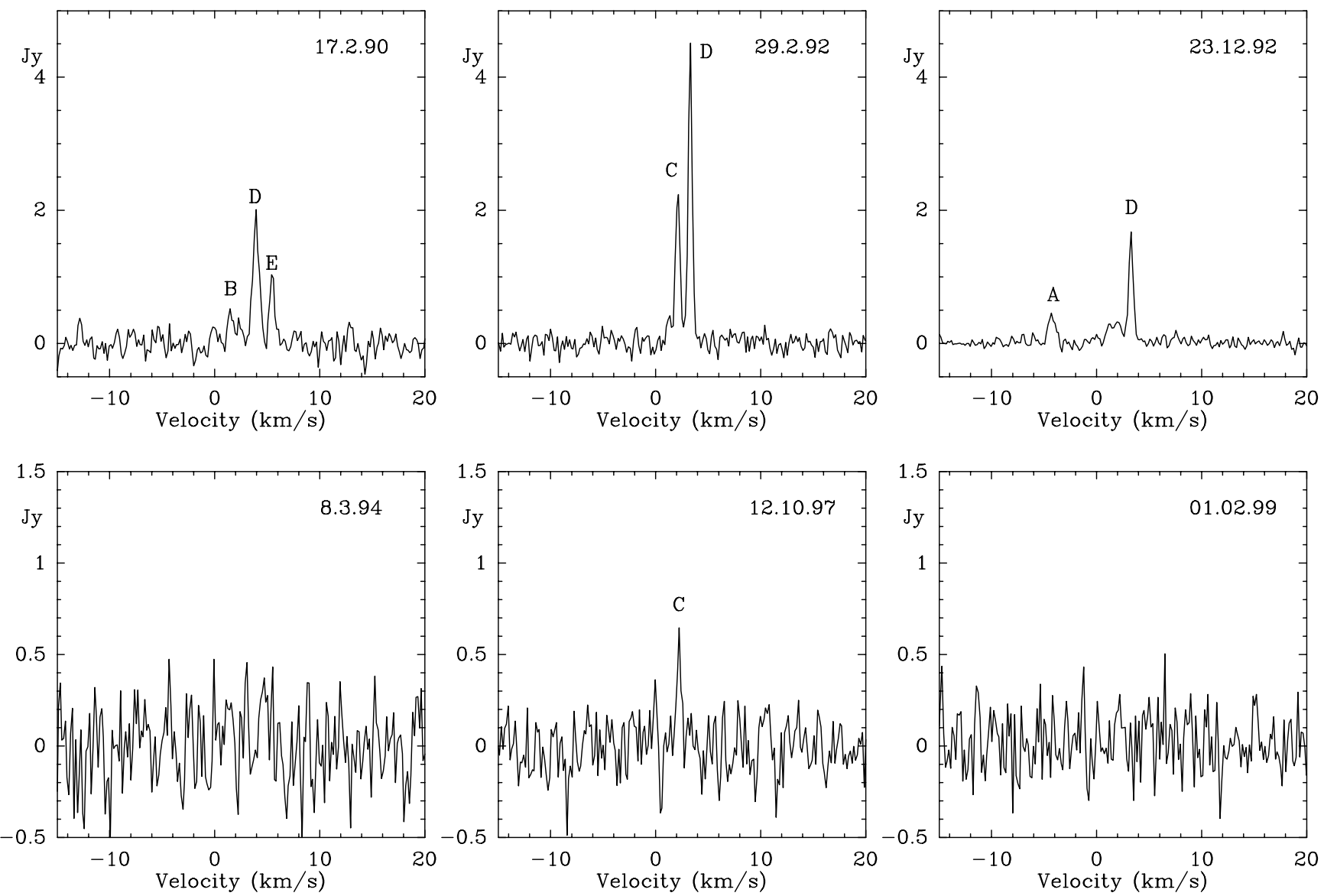

Fig. 1. Representative $22 \mathrm{GHz} \mathrm{H}_{2} \mathrm{O}$ maser spectra of $\mathrm{OH} 15.7+0.8$. Maser components listed in Table 2 are labeled.

Table 2. Maser components of $\mathrm{OH} 15.7+0.8$. The integrated flux $S_{\mathrm{I}}$ is given in $10^{-22}$ Watt $^{-2}$.

\begin{tabular}{|c|c|c|c|c|c|c|c|c|c|c|c|c|c|}
\hline \multirow[b]{2}{*}{ Date } & \multirow[b]{2}{*}{ JD } & \multirow[b]{2}{*}{$\begin{array}{l}\mathrm{rms} \\
{[\mathrm{Jy}]}\end{array}$} & \multirow[b]{2}{*}{$S_{\text {I }}$} & \multicolumn{2}{|c|}{$\mathrm{A}$} & \multicolumn{2}{|c|}{ B } & \multicolumn{2}{|c|}{$\mathrm{C}$} & \multicolumn{2}{|c|}{$\mathrm{D}$} & \multicolumn{2}{|c|}{$\mathrm{E}$} \\
\hline & & & & $v_{\mathrm{p}}$ & $S_{\mathrm{p}}$ & $v_{\mathrm{p}}$ & $S_{\mathrm{p}}$ & $v_{\mathrm{p}}$ & $\begin{array}{c}S_{\mathrm{p}} \\
\mathrm{Jy}]\end{array}$ & $v_{\mathrm{p}}$ & $S_{\mathrm{p}}$ & $v_{\mathrm{p}}$ & $S_{\mathrm{p}}$ \\
\hline 17.02 .90 & 2447940 & 0.16 & 17: & - & - & 1.5 & 0.6 & 2.4 & 0.3: & 3.9 & 2.1 & 5.5 & 1.1 \\
\hline 01.04 .90 & 2447983 & 0.30 & 32 & - & - & - & - & - & - & 3.9 & 2.5 & 5.4 & 2.1 \\
\hline 01.05 .91 & 2448378 & 0.36 & 16 & - & - & - & - & 2.2 & 1.8 & 3.5 & 2.7 & - & - \\
\hline 29.02.92 & 2448682 & 0.19 & 27 & - & - & 1.3 & 0.3: & 2.1 & 2.4 & 3.3 & 4.8 & - & - \\
\hline 23.12 .92 & 2448980 & 0.10 & 14 & -4.3 & 0.5 & 1.3 & 0.3 & 2.1 & 0.3 & 3.3 & 1.8 & - & - \\
\hline 20.04 .93 & 2449098 & 0.20 & 10 & -4.2 & 0.5 & 1.3 & 0.3 : & 2.1 & 0.5 : & 3.3 & 1.9 & - & - \\
\hline 08.03 .94 & 2449420 & 0.30 & $<10$ & - & - & - & - & - & - & - & - & - & - \\
\hline 10.03 .95 & 2449786 & 0.14 & $<10$ & - & - & - & - & - & - & - & - & - & - \\
\hline 03.09 .96 & 2450330 & 0.22 & $<10$ & - & - & - & - & - & - & - & - & - & - \\
\hline 12.10 .97 & 2450734 & 0.13 & $<10$ & - & - & - & - & 2.3 & 0.6 & - & - & - & - \\
\hline 01.02 .99 & 2451211 & 0.26 & $<10$ & - & - & - & - & - & - & - & - & - & - \\
\hline
\end{tabular}

is unique, because the most blueshifted line at $\approx-6 \mathrm{~km} \mathrm{~s}^{-1}$ was never detected before or afterwards in Effelsberg spectra. After 1990, our monitor program detected maser lines with similar intensities (Fig. 1) in a smaller velocity range between -4.3 and $+5.5 \mathrm{~km} \mathrm{~s}^{-1}$ (Table 2 ).

Adopting $-7 \mathrm{~km} \mathrm{~s}^{-1}$ from the 1986 December observation of Benson \& Little-Marenin as the blue edge of the velocity interval and $+6.3 \mathrm{~km} \mathrm{~s}^{-1}$ as the red edge, we find a center of the range $v_{\mathrm{c}}^{\mathrm{H}_{2} O}=-0.3 \mathrm{~km} \mathrm{~s}^{-1}$ consistent with the center of the $1612 \mathrm{MHz} \mathrm{OH}$ maser velocity interval at $-0.6 \mathrm{~km} \mathrm{~s}^{-1}$ (Herman et al. 1985). Taking the latter as the radial velocity $v_{\mathrm{c}}$ of the central star, an $\mathrm{H}_{2} \mathrm{O}$ outflow velocity of $v_{\mathrm{e}}^{\mathrm{H}_{2} \mathrm{O}} \approx 6.5 \mathrm{~km} \mathrm{~s}^{-1}$ (Table 1 ) is inferred.

In our monitoring spectra we identified 5 maser components (Table 2). As before 1990, the bulk of the emission comes from velocities redshifted with respect to the radial velocity, with the strongest component D at 3.3$3.9 \mathrm{~km} \mathrm{~s}^{-1}$. In 1990, the velocity of D was definitely larger 

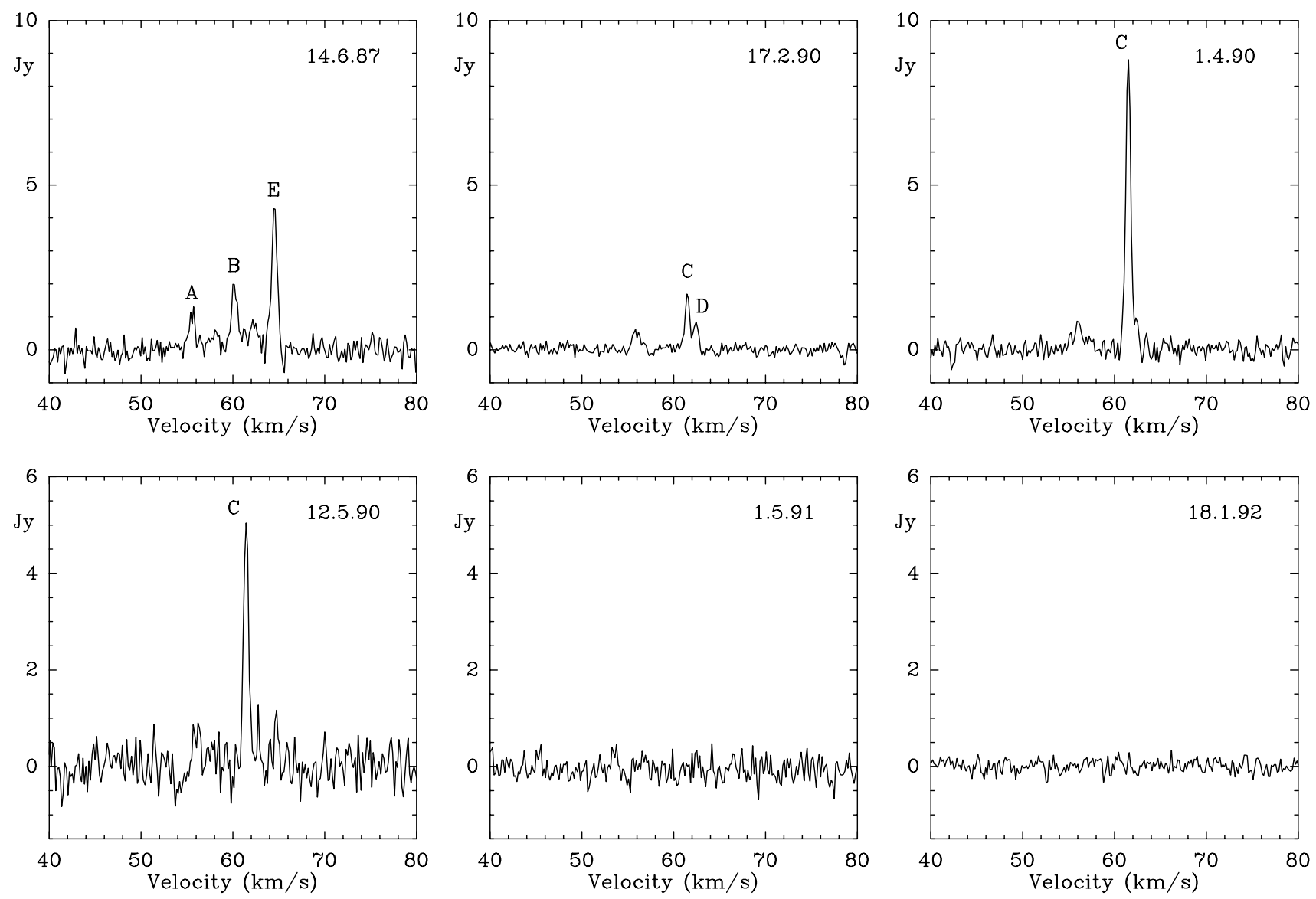

Fig. 2. $\mathrm{H}_{2} \mathrm{O}$ maser spectra of $\mathrm{OH}$ 17.7-2.0 1987-1992. Maser components listed in Table 3 are labeled. Between 1993 and 1999 no maser was detected (cf. Table 3).

Table 3. Maser components of $\mathrm{OH} 17.7-2.0$. The integrated flux $S_{\mathrm{I}}$ is given in $10^{-22}$ Watt $\mathrm{m}^{-2}$.

\begin{tabular}{|c|c|c|c|c|c|c|c|c|c|c|c|c|c|}
\hline \multirow[b]{2}{*}{ Date } & \multirow[b]{2}{*}{ JD } & \multirow[b]{2}{*}{$\begin{array}{l}\mathrm{rms} \\
{[\mathrm{Jy}]}\end{array}$} & \multirow[b]{2}{*}{$S_{\text {I }}$} & \multicolumn{2}{|c|}{$\mathrm{A}$} & \multicolumn{2}{|c|}{ B } & \multicolumn{2}{|c|}{$\mathrm{C}$} & \multicolumn{2}{|c|}{$\mathrm{D}$} & \multicolumn{2}{|c|}{$\mathrm{E}$} \\
\hline & & & & $v_{\mathrm{p}}$ & $S_{\mathrm{p}}$ & $v_{\mathrm{p}}$ & $S_{\mathrm{p}}$ & $\begin{array}{c}v_{\mathrm{p}} \\
{[\mathrm{km} \mathrm{s}}\end{array}$ & $\begin{array}{l}S_{\mathrm{p}} \\
1, \mathrm{Jy}\end{array}$ & $v_{\mathrm{p}}$ & $S_{\mathrm{p}}$ & $v_{\mathrm{p}}$ & $S_{\mathrm{p}}$ \\
\hline 14.06 .87 & 2446961 & 0.39 & 60 & 55.6 & 1.2 & 60.2 & 2.1 & - & - & 62.1 & $0.8:$ & 64.6 & 4.4 \\
\hline 17.02 .90 & 2447940 & 0.18 & 13: & 56.0 & 0.5 & - & - & 61.5 & 1.8 & 62.4 & 0.8 & - & - \\
\hline 01.04 .90 & 2447983 & 0.31 & 58 & 56.1 & 0.6 & - & - & 61.5 & 9.2 & 62.4 & 1.0 & - & - \\
\hline 12.05 .90 & 2448024 & 0.56 & 37 & - & - & - & - & 61.5 & 5.3 & - & - & - & - \\
\hline 01.05 .91 & 2448378 & 0.35 & $<10$ & - & - & - & - & - & - & - & - & - & - \\
\hline 18.01 .92 & 2448640 & 0.21 & $<10$ & - & - & - & - & - & - & - & - & - & - \\
\hline 22.12 .92 & 2448979 & 0.33 & $<10$ & - & - & - & - & - & - & - & - & - & - \\
\hline 08.03 .94 & 2449420 & 0.31 & $<10$ & - & - & - & - & - & - & - & - & - & - \\
\hline 09.03 .95 & 2449786 & 0.15 & $<10$ & - & - & - & - & - & - & - & - & - & - \\
\hline 03.09 .96 & 2450330 & 0.23 & $<10$ & - & - & - & - & - & - & - & - & - & - \\
\hline 12.10 .97 & 2450734 & 0.23 & $<10$ & - & - & - & - & - & - & - & - & - & - \\
\hline 01.02 .99 & 2451211 & 0.26 & $<10$ & - & - & - & - & - & - & - & - & - & - \\
\hline
\end{tabular}

than in $1992 / 1993$. This behaviour is probably not due to a velocity shift of one line, but due to the presence of two lines with slightly different velocities and typical lifetimes of 1-2 years. Similarly the velocity shift of component C might be explained. The widths of the stronger lines are
$0.4-0.8 \mathrm{~km} \mathrm{~s}^{-1}$, and intensity variations up to a factor of two were observed for individual maser lines on the time scale of months. After 1993 the maser was redetected only once in 1997. A non-detection ( $\mathrm{rms} \approx 1 \mathrm{Jy})$ was reported for 1992 August also by Benson \& Little-Marenin (1996). 
Table 4. Blue maser components of $\mathrm{OH} 12.8-0.9 . S_{\mathrm{I}}^{\text {blue }}$ is the integrated flux between -84 and $-58 \mathrm{~km} \mathrm{~s}^{-1}{\mathrm{in} 10^{-22} \mathrm{Wm}}^{-2}$.

\begin{tabular}{|c|c|c|c|c|c|c|c|c|c|c|c|c|c|}
\hline \multirow[b]{2}{*}{ Date } & \multirow[b]{2}{*}{ JD } & \multirow[b]{2}{*}{$\begin{array}{l}\mathrm{rms} \\
{[\mathrm{Jy}]}\end{array}$} & \multirow[b]{2}{*}{$S_{\mathrm{I}}^{\text {blue }}$} & \multicolumn{2}{|c|}{$\mathrm{A}$} & \multicolumn{2}{|c|}{ B } & \multicolumn{2}{|c|}{$\mathrm{C}$} & \multicolumn{2}{|c|}{$\mathrm{D}$} & \multicolumn{2}{|c|}{$\mathrm{E}$} \\
\hline & & & & $v_{\mathrm{p}}$ & $S_{\mathrm{p}}$ & $v_{\mathrm{p}}$ & $S_{\mathrm{p}}$ & $\begin{array}{c}v_{\mathrm{p}} \\
-\left[\mathrm{km} \mathrm{s}^{-}\right.\end{array}$ & $\begin{array}{l}S_{\mathrm{p}} \\
\left.{ }^{1}, \mathrm{Jy}\right]\end{array}$ & $v_{\mathrm{p}}$ & $S_{\mathrm{p}}$ & $v_{\mathrm{p}}$ & $S_{\mathrm{p}}$ \\
\hline 14.06 .87 & 2446961 & 0.21 & 169 & - & - & - & - & - & - & - & - & -76.4 & 9.2 \\
\hline 07.07 .88 & 2447350 & 0.36 & 191 & - & - & - & - & - & - & - & - & -76.6 & 11.9 \\
\hline 17.02 .90 & 2447940 & 0.33 & 97 & -81.8 & 1.4 & - & - & -78.7 & 3.1 & -77.9 & 3.6 & -76.8 & 5.1 \\
\hline 01.04 .90 & 2447983 & 0.46 & 191 & -81.9 & 1.9 & - & - & -78.9 & 5.0 & -77.9 & 4.8 & -76.9 & 10.0 \\
\hline 01.05 .91 & 2448378 & 0.42 & 178 & -81.6 & 1.1 & -79.8 & 3.6 & - & - & - & - & -76.9 & 13.0 \\
\hline 18.01 .92 & 2448640 & 0.32 & 141 & - & - & -80.0 & 8.9 & - & - & -77.9 & 5.1 & -77.1 & 5.3 \\
\hline 22.12 .92 & 2448979 & 0.48 & 138 & -82.4 & 1.4: & -80.1 & 5.1 & - & - & -78.1 & 1.8 & -77.1 & 3.6 \\
\hline 08.03 .94 & 2449420 & 0.37 & 95 & - & - & -80.1 & 3.2 & - & - & - & - & -77.3 & 7.3 \\
\hline 09.03 .95 & 2449786 & 0.19 & 142 & - & - & -80.2 & 9.1 & -79.4 & 2.7: & - & - & -77.5 & 10.3 \\
\hline 03.09 .96 & 2450330 & 0.22 & 27 & - & - & -80.6 & 2.0 & -78.9 & 0.7 & - & - & -77.5 & 2.2 \\
\hline \multirow[t]{2}{*}{01.02 .99} & 2451211 & 0.27 & 28 & -82.7 & 1.4 & -81.0 & 0.9 & - & - & - & - & -77.6 & 1.0: \\
\hline & & & & \multicolumn{2}{|c|}{$\mathrm{F}$} & \multicolumn{2}{|c|}{ G } & \multicolumn{2}{|c|}{$\mathrm{H}$} & \multicolumn{2}{|c|}{ I } & & \\
\hline Date & JD & $\begin{array}{l}\mathrm{rms} \\
{[\mathrm{Jy}]}\end{array}$ & & $v_{\mathrm{p}}$ & $S_{\mathrm{p}}$ & $v_{\mathrm{p}}$ & $\begin{array}{l}S_{\mathrm{p}} \\
{\left[\mathrm{kms}^{-}\right.}\end{array}$ & $\begin{array}{c}v_{\mathrm{p}} \\
1, \mathrm{Jy}]\end{array}$ & $S_{\mathrm{p}}$ & $v_{\mathrm{p}}$ & $S_{\mathrm{p}}$ & & \\
\hline 14.06 .87 & 2446961 & 0.21 & & -75.0 & 1.7 & -73.3 & 4.5 & - & - & - & - & & \\
\hline 07.07 .88 & 2447350 & 0.36 & & -75.3 & 1.9 & -73.2 & 5.5 & -71.1 & 2.0 & - & - & & \\
\hline 17.02 .90 & 2447940 & 0.33 & & -75.8 & 2.1 & - & - & -70.9 & 0.9: & -63.6 & 0.9 : & & \\
\hline 01.04 .90 & 2447983 & 0.46 & & -75.7 & 3.3 & - & - & -70.9 & 1.3 & -63.6 & 1.7 & & \\
\hline 01.05 .91 & 2448378 & 0.42 & & -75.4 & 6.3 & -73.7 & 3.8 & -71.0 & 1.3 & -63.7 & 0.7 : & & \\
\hline 18.01 .92 & 2448640 & 0.32 & & -75.5 & 1.1 & - & - & - & - & - & - & & \\
\hline 22.12 .92 & 2448979 & 0.48 & & -75.2 & 2.4 & - & - & - & - & - & - & & \\
\hline 08.03 .94 & 2449420 & 0.37 & & -76.4 & 2.7 & - & - & - & - & - & - & & \\
\hline 09.03 .95 & 2449786 & 0.19 & & -76.3 & 1.8 & - & - & - & - & - & - & & \\
\hline 03.09.96 & 2450330 & 0.22 & & - & - & - & - & - & - & - & - & & \\
\hline 01.02 .99 & 2451211 & 0.27 & & - & - & - & - & - & - & - & - & & \\
\hline
\end{tabular}

\section{2. $\mathrm{OH} 17.7-2.0$}

The $\mathrm{H}_{2} \mathrm{O}$ maser of $\mathrm{OH} 17.7-2.0$ has undergone a dramatic decrease in intensity since its discovery in 1983 by Nyman et al. (1986). Until 1985 the strongest maser line always had a flux density $>50 \mathrm{Jy}$ (Engels et al. 1986), and dropped 1987 to a level a magnitude lower (Likkel 1989, Table 3). Our observations show that after 1990 the maser disappeared completely (Fig. 2).

Engels et al. (1986) found an $\mathrm{H}_{2} \mathrm{O}$ maser velocity range of $\Delta v=10.2 \mathrm{~km} \mathrm{~s}^{-1}$ (full width measured at zero intensity $(F W Z I)$ ) between 52.8 and $63.0 \mathrm{~km} \mathrm{~s}^{-1}$. In 1987 a line was detected close to $65 \mathrm{~km} \mathrm{~s}^{-1}$ (Likkel 1989, Table 3 component $\mathrm{E}$ ), increasing the range with observed maser lines to $\Delta v=12.2 \mathrm{~km} \mathrm{~s}^{-1}$. The radial velocity of the star is $61.4 \pm 0.2 \mathrm{~km} \mathrm{~s}^{-1}$, as judged from $\mathrm{OH}$ and $\mathrm{CO}$ measurements (Herman et al. 1985; Heske et al. 1990), implying an $\mathrm{H}_{2} \mathrm{O}$ outflow velocity of $v_{\mathrm{e}}^{\mathrm{H}_{2} \mathrm{O}}=8.6 \mathrm{~km} \mathrm{~s}^{-1}$ (Table 1 ). Before 1987 most of the $\mathrm{H}_{2} \mathrm{O}$ maser emission was emitted bluewards of the radial velocity, in 1987 the strongest component $\mathrm{E}$ was emitted redwards, and in 1990 the main peak (component $\mathrm{C}$ ) coincided with the radial velocity.

Judging on the velocities, the lines seen in 1984/85 (cf. Engels et al. 1986), 1987 and 1990 were different in each epoch. The formation of new maser lines after $2-3$ years corroborates the observations of $\mathrm{OH} 15.7+0.8$, that the lifetime of the maser clouds is limited and of the order of $\$ 3$ years. Only one of the strong (>50 Jy) lines seen in 1984/85 was redetected later on. In 1985 this line at $61.5 \mathrm{~km} \mathrm{~s}^{-1}$ had $\approx 100 \mathrm{Jy}$, it was weak or absent in 1987 $(<1 \mathrm{Jy})$ and reached in $1990 \approx 9 \mathrm{Jy}$. Possibly also this maser line corresponded in 1990 to a different component than in $1984 / 85$.

In each epoch, in which the $\mathrm{H}_{2} \mathrm{O}$ maser of $\mathrm{OH} 17.7-2.0$ was observed two or three times, the individual maser lines displayed intensity variations by factors $2-5$ over a few months. Half widths of the observed maser lines were $0.6-0.8 \mathrm{~km} \mathrm{~s}^{-1}$, except for component A with a $F W H M$ of $0.8-1.7 \mathrm{~km} \mathrm{~s}^{-1}$. The large half widths and the velocity shift from 1987 to 1990 indicate that component A is a blend of at least two lines (see also Fig. 2).

\section{3. $\mathrm{OH} 12.8-0.9$}

While the previous two sources showed $\mathrm{H}_{2} \mathrm{O}$ maser emission in the vicinity of the radial velocity of the star, the following two sources have their main emission features outside the velocity interval defined by the $\mathrm{OH}$ maser emission. OH 12.8-0.9 was discovered by Engels et al. (1986) displaying two groups of several maser lines at the 
D. Engels: Water vapor masers in stars departing from the AGB
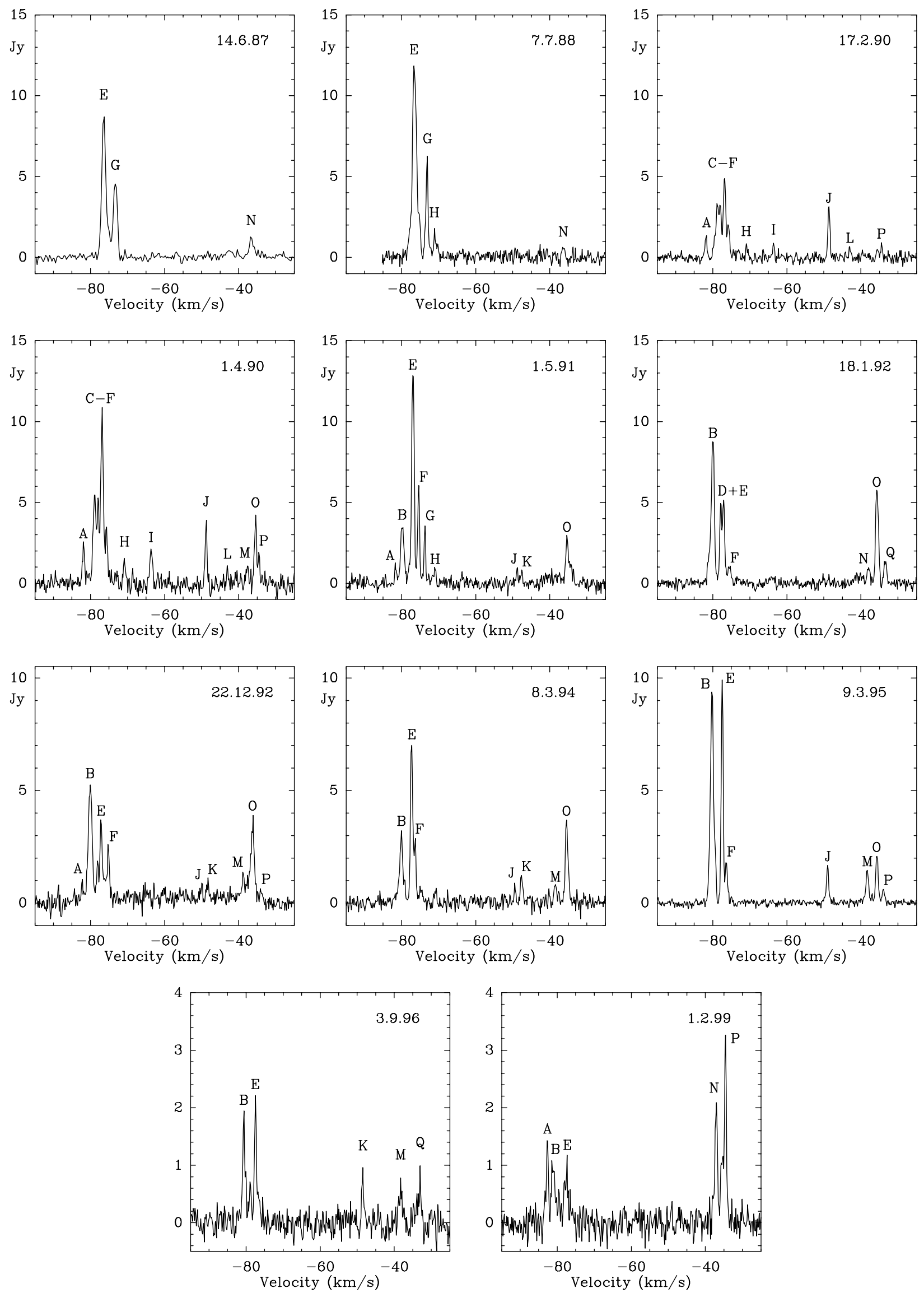

Fig. 3. $\mathrm{H}_{2} \mathrm{O}$ maser spectra of $\mathrm{OH} 12.8-0.9$. Maser components listed in Tables 4 and 5 are labeled. 


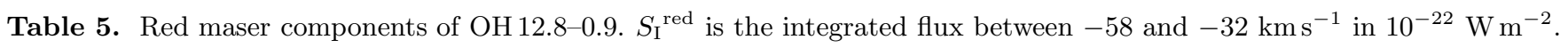

\begin{tabular}{|c|c|c|c|c|c|c|c|c|c|c|c|c|c|}
\hline \multirow[b]{2}{*}{ Date } & \multirow[b]{2}{*}{ JD } & \multirow[b]{2}{*}{$\begin{array}{l}\mathrm{rms} \\
{[\mathrm{Jy}]}\end{array}$} & \multirow[b]{2}{*}{$S_{\mathrm{I}}^{\text {red }}$} & \multicolumn{2}{|c|}{$\mathrm{J}$} & \multicolumn{2}{|c|}{$\mathrm{K}$} & \multicolumn{2}{|c|}{$\mathrm{L}$} & \multicolumn{2}{|c|}{ M } & \multicolumn{2}{|c|}{$\mathrm{N}$} \\
\hline & & & & $v_{\mathrm{p}}$ & $S_{\mathrm{p}}$ & $v_{\mathrm{p}}$ & $S_{\mathrm{p}}$ & $\begin{array}{c}v_{\mathrm{p}} \\
{\left[\mathrm{km} \mathrm{s}^{-}\right.}\end{array}$ & $\begin{array}{c}S_{\mathrm{p}} \\
1, \mathrm{Jy}]\end{array}$ & $v_{\mathrm{p}}$ & $S_{\mathrm{p}}$ & $v_{\mathrm{p}}$ & $S_{\mathrm{p}}$ \\
\hline 14.06 .87 & 2446961 & 0.21 & 65 & - & - & - & - & - & - & - & - & -36.6 & 1.1 \\
\hline 07.07 .88 & 2447350 & 0.36 & 12 & - & - & - & - & - & - & - & - & -36.4 & 0.6 : \\
\hline 17.02 .90 & 2447940 & 0.33 & 21 & -48.7 & 3.3 & - & - & -43.1 & $0.8:$ & - & - & - & - \\
\hline 01.04 .90 & 2447983 & 0.46 & 47 & -48.8 & 3.8 & - & - & -43.1 & 1.1: & -37.7 & 1.0 & - & - \\
\hline 01.05 .91 & 2448378 & 0.42 & 45 & -48.8 & 0.9: & -47.5 & 0.8 & - & - & - & - & - & - \\
\hline 18.01 .92 & 2448640 & 0.32 & 67 & - & - & - & - & - & - & -37.9 & 1.0 & - & - \\
\hline 22.12 .92 & 2448979 & 0.48 & 92 & -49.9 & $0.8:$ & -48.3 & $0.8:$ & - & - & -38.8 & 1.3 & - & - \\
\hline 08.03 .94 & 2449420 & 0.37 & 51 & -49.4 & $0.8:$ & -47.6 & 1.2 & - & - & -38.6 & 0.8 & - & - \\
\hline 09.03 .95 & 2449786 & 0.19 & 42 & -49.0 & 1.7 & - & - & - & - & -38.3 & 1.5 & - & - \\
\hline 03.09 .96 & 2450330 & 0.22 & 14 & - & - & -48.5 & 0.9 & - & - & -38.3 & 0.8 & - & - \\
\hline \multirow[t]{2}{*}{01.02 .99} & 2451211 & 0.27 & 39 & - & - & - & - & - & - & - & - & -37.1 & 2.0 \\
\hline & & & & \multicolumn{2}{|c|}{$\mathrm{O}$} & \multicolumn{2}{|c|}{$\mathrm{P}$} & \multicolumn{2}{|c|}{$\mathrm{Q}$} & & & & \\
\hline Date & JD & \multicolumn{2}{|l|}{$\begin{array}{l}\mathrm{rms} \\
{[\mathrm{Jy}]}\end{array}$} & \multicolumn{2}{|r|}{$S_{\mathrm{p}}$} & \multicolumn{2}{|c|}{$v_{\mathrm{p}} \quad S_{\mathrm{p}}$} & $v_{\mathrm{p}}$ & $S_{\mathrm{p}}$ & & & & \\
\hline 14.06 .87 & 2446961 & 0.21 & & -35.7 & 0.4: & - & - & - & - & & & & \\
\hline 07.07 .88 & 2447350 & 0.36 & & - & - & - & - & - & - & & & & \\
\hline 17.02 .90 & 2447940 & 0.33 & & -35.5 & 0.4: & -34.4 & 0.8 & - & - & & & & \\
\hline 01.04 .90 & 2447983 & 0.46 & & -35.4 & 3.9 & -34.5 & 1.9 & - & - & & & & \\
\hline 01.05 .91 & 2448378 & 0.42 & & -35.4 & 2.7 & -34.4 & 1.0 & - & - & & & & \\
\hline 18.01 .92 & 2448640 & 0.32 & & -35.7 & 6.0 & - & - & -33.4 & 1.4 & & & & \\
\hline 22.12 .92 & 2448979 & 0.48 & & -36.2 & 4.3 & -34.0 & 0.5 : & - & - & & & & \\
\hline 08.03 .94 & 2449420 & 0.37 & & -35.4 & 3.6 & - & - & - & - & & & & \\
\hline 09.03 .95 & 2449786 & 0.19 & & -35.7 & 2.2 & -34.0 & 0.6 & - & - & & & & \\
\hline 03.09 .96 & 2450330 & 0.22 & & - & - & - & - & -33.2 & 1.0 & & & & \\
\hline 01.02 .99 & 2451211 & 0.27 & & -35.5 & 1.2 & -34.6 & 3.2 & - & - & & & & \\
\hline
\end{tabular}

ends of a $38.7 \mathrm{~km} \mathrm{~s}^{-1}$ wide velocity interval, which was centered approximately on the radial velocity of the star, as given by the $\mathrm{OH}$ maser. This $\mathrm{H}_{2} \mathrm{O}$ maser was unique among the $\mathrm{OH} / \mathrm{IR}$ stars studied, showing an $\mathrm{H}_{2} \mathrm{O}$ outflow velocity surpassing the $\mathrm{OH}$ outflow velocity by several $\mathrm{kms}^{-1}$. Gómez et al. (1994) showed that the masers coincide positionally within $1^{\prime \prime}$, and belong therefore to the same object. Between 1987 and 1999 (Fig. 3) the overall spectral shape and the intensities remained similar to the earlier spectra, but additional weaker lines were discovered at velocities in between the main groups. The components identified in the monitoring spectra are given in Tables 4 and 5 . The blueshifted group of components cover velocities $-83<v<-70 \mathrm{~km} \mathrm{~s}^{-1}$ (components A$\mathrm{H})$ and the redshifted group $-39<v<-33 \mathrm{kms}^{-1}$ (components $\mathrm{M}-\mathrm{Q}$ ). In between lines were assigned to four components (I-L).

Due to blending the assignment of the lines to the different components is difficult for this source in particular. The half widths of the maser lines range between 0.4 and $1.2 \mathrm{~km} \mathrm{~s}^{-1}$. We suspect that lines showing $F W H M>0.8 \mathrm{~km} \mathrm{~s}^{-1}$ are affected by blending. The strong velocity shifts of $\approx 1 \mathrm{~km} \mathrm{~s}^{-1}$ of several components (f.e. B, E, F and K) are therefore more likely caused by relative changes of intensities within blended features than due to velocity shifts of a single component. The true number of components present is probably substantially larger than the 17 components listed in Tables 4 and 5. Except for the components with the apparent velocity shifts, components were detected consecutively at most over 3 years. The lifetimes of the emitting clouds are definitely shorter than the interval covered by the observations and are probably between one and three years.

With respect to the observations before 1987 the spectra reveal an increase of the $\mathrm{H}_{2} \mathrm{O}$ maser velocity range. The total width is now $\Delta v=50 \mathrm{~km} \mathrm{~s}^{-1}(F W Z I)$ between $\approx-83 \mathrm{~km} \mathrm{~s}^{-1}$ and $\approx-33 \mathrm{~km} \mathrm{~s}^{-1}$. The radial velocity of the star derived from the $\mathrm{OH}$ maser emission is $v_{\mathrm{c}}=-55.5 \pm 0.5 \mathrm{~km} \mathrm{~s}^{-1}$ (Baud et al. 1979; Dickinson \& Turner 1991), inferring an $\mathrm{H}_{2} \mathrm{O}$ outflow velocity of $v_{\mathrm{e}}^{\mathrm{H}_{2} \mathrm{O}} \approx 28 \mathrm{~km} \mathrm{~s}^{-1}$ (Table 1 ). The emission at the most extreme velocities between -82 and $-77 \mathrm{~km} \mathrm{~s}^{-1}$ and between -35 and $-33 \mathrm{~km} \mathrm{~s}^{-1}$ appeared only after 1988 , and this increase of the $\mathrm{H}_{2} \mathrm{O}$ velocity range since 1989 is considered to be real and not an artifact of improved sensitivity for the later observations. 

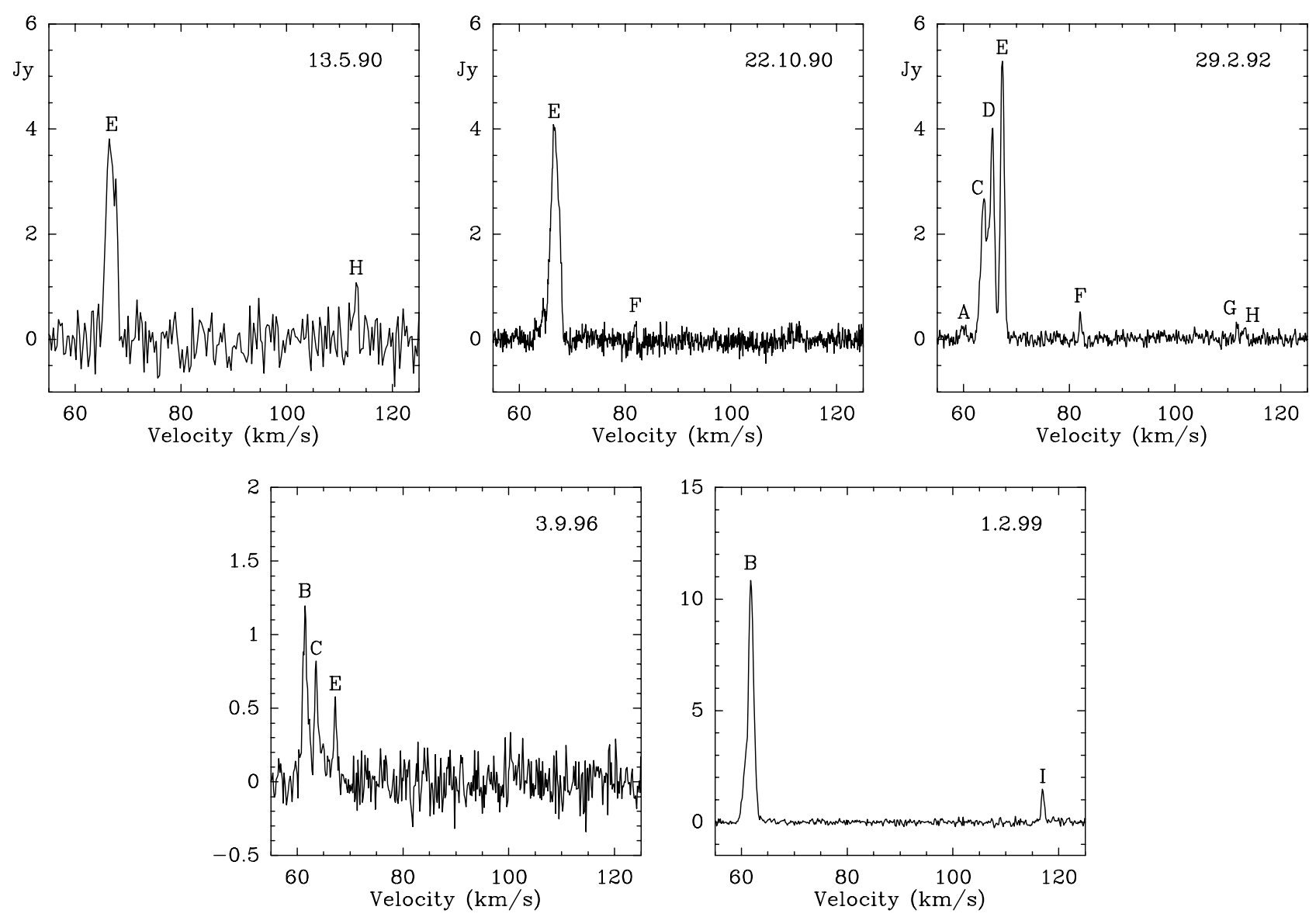

Fig. 4. $\mathrm{H}_{2} \mathrm{O}$ maser spectra of $\mathrm{OH} 37.1-0.8$. Maser components listed in Table 6 are labeled.

Table 6. Maser components of $\mathrm{OH} 37.1-0.8$. The integrated flux $S_{\mathrm{I}}$ is given in $10^{-22} \mathrm{~W} \mathrm{~m}^{-2}$.

\begin{tabular}{|c|c|c|c|c|c|c|c|c|c|c|c|c|c|}
\hline \multirow[b]{2}{*}{ Date } & \multirow[b]{2}{*}{$\mathrm{JD}$} & \multirow[b]{2}{*}{$\begin{array}{l}\mathrm{rms} \\
{[\mathrm{Jy}]}\end{array}$} & \multirow[b]{2}{*}{$S_{\text {I }}$} & \multicolumn{2}{|c|}{$\mathrm{A}$} & \multicolumn{2}{|c|}{ B } & \multicolumn{2}{|c|}{$\mathrm{C}$} & \multicolumn{2}{|c|}{$\mathrm{D}$} & \multicolumn{2}{|c|}{$\mathrm{E}$} \\
\hline & & & & $v_{\mathrm{p}}$ & $S_{\mathrm{p}}$ & $v_{\mathrm{p}}$ & $S_{\mathrm{p}}$ & $\begin{array}{c}v_{\mathrm{p}} \\
{\left[\mathrm{km} \mathrm{s}^{-1}\right.}\end{array}$ & $\begin{array}{l}S_{\mathrm{p}} \\
, \mathrm{Jy}]\end{array}$ & $v_{\mathrm{p}}$ & $S_{\mathrm{p}}$ & $v_{\mathrm{p}}$ & $S_{\mathrm{p}}$ \\
\hline 13.05 .90 & 2448025 & 0.29 & 66 & - & - & - & - & - & - & - & - & 66.8 & 3.8 \\
\hline 22.10 .90 & 2448187 & 0.12 & 51 & - & - & - & - & 63.1 & $0.3:$ & 64.5 & 0.6 : & 66.7 & 4.0 \\
\hline 29.02 .92 & 2448682 & 0.09 & 101 & 59.7 & 0.2 : & - & - & 63.9 & 2.6 & 65.5 & 3.8 & 67.4 & 5.4 \\
\hline 03.09 .96 & 2450330 & 0.19 & 20 & - & - & 61.5 & 1.4 & 63.6 & 0.8 & - & - & 67.2 & 0.6 \\
\hline \multirow[t]{2}{*}{01.02 .99} & 2451211 & 0.13 & 122 & 60.5 & 2.0 & 61.8 & 10.7 & - & - & - & - & - & - \\
\hline & & & & \multicolumn{2}{|c|}{$\mathrm{F}$} & \multicolumn{2}{|c|}{$G$} & \multicolumn{2}{|c|}{$\mathrm{H}$} & \multicolumn{2}{|c|}{$\mathrm{I}$} & & \\
\hline Date & JD & $\begin{array}{l}\mathrm{rms} \\
{[\mathrm{Jy}]}\end{array}$ & & $v_{\mathrm{p}}$ & $S_{\mathrm{p}}$ & $v_{\mathrm{p}}$ & $\begin{array}{c}S_{\mathrm{p}} \\
-[\mathrm{kms}\end{array}$ & $\begin{array}{c}v_{\mathrm{p}} \\
1, \mathrm{Jy}]\end{array}$ & $S_{\mathrm{p}}$ & $v_{\mathrm{p}}$ & $S_{\mathrm{p}}$ & & \\
\hline 13.05 .90 & 2448025 & 0.29 & & - & - & - & - & 113.2 & 1.1 & - & - & & \\
\hline 22.10 .90 & 2448187 & 0.12 & & 81.9 & $0.3:$ & 111.4 & 0.2 : & 112.8 & 0.2 : & - & - & & \\
\hline 29.02 .92 & 2448682 & 0.09 & & 82.1 & 0.5 & 111.7 & 0.3 & 113.1 & 0.2 & - & - & & \\
\hline 03.09 .96 & 2450330 & 0.19 & & - & - & - & - & - & - & - & - & & \\
\hline 01.02.99 & 2451211 & 0.13 & & - & - & - & - & - & - & 117.0 & 1.5 & & \\
\hline
\end{tabular}

\section{4. $\mathrm{OH} 37.1-0.8$}

The water vapor maser of OH 37.1-0.8 was discovered by Engels et al. (1986), but was not considered to be associated with the star, because the blueshifted emission was outside the $\mathrm{OH}$ maser velocity range and the redshifted emission was not seen. An early attempt to detect $\mathrm{H}_{2} \mathrm{O}$ maser emission by Cato et al. (1976) failed. Their detection limit in 1974 was $\approx 2 \mathrm{Jy}(3 \sigma)$. Gómez et al. (1994) showed that the $\mathrm{H}_{2} \mathrm{O}$ maser emission is positionally coincident with the $\mathrm{OH}$ maser emission within $1^{\prime \prime}$ and they detected the redshifted emission in 1990, proving the 
association of the $\mathrm{H}_{2} \mathrm{O}$ maser with $\mathrm{OH} 37.1-0.8$. We found the redshifted emission lines independently in 1990 and the high signal-to-noise ratio spectrum taken in 1992 shows that $\mathrm{OH} 37.1-0.8$ has at least 7 individual maser components (Fig. 4, Table 6). OH 37.1-0.8 was not observed frequently enough to determine the lifetime of the maser lines. Nevertheless the velocities vary from epoch to epoch considerably, being compatible with a mean lifetime of the emitting clouds of a few years.

In 1999 we detected a line at $117.0 \mathrm{~km} \mathrm{~s}^{-1}$ (component I) outside the velocity range observed so far. As for $\mathrm{OH} 12.8-0.9$ the velocity range covered by the $\mathrm{H}_{2} \mathrm{O}$ masers seems to increase with time. From the 1999 spectrum we derive a current $\mathrm{H}_{2} \mathrm{O}$ velocity range of $\Delta v \approx 57 \mathrm{~km} \mathrm{~s}^{-1}$ with the midpoint at $88.5 \mathrm{~km} \mathrm{~s}^{-1}$ in perfect agreement with the radial velocity given by the $\mathrm{OH}$ maser (Table 1 ). The corresponding maximum $\mathrm{H}_{2} \mathrm{O}$ outflow velocity is $v_{\mathrm{e}}^{\mathrm{H}_{2} \mathrm{O}}=28.5 \mathrm{~km} \mathrm{~s}^{-1}$, which is about twice the expansion velocity of the $\mathrm{OH}$ emitting shell $\left(v_{\mathrm{e}}=14.9 \mathrm{~km} \mathrm{~s}^{-1}\right)$.

\subsection{Summary: common characteristics of the $\mathrm{H}_{2} \mathrm{O}$ maser spectra}

\subsubsection{Blending and velocity shifts}

The water vapor masers of the sources described here consist typically of many individual maser lines, which show up in the maser spectra as distinct features, if there is sufficient dispersion in their projected velocities. We identified 5 to 17 different components, which in part are blends of lines with velocity differences $<1 \mathrm{~km} \mathrm{~s}^{-1}$. The presence of significant blending is evident from the asymmetry of particular lines and the presence of velocity shifts of the order $\leq 1 \mathrm{~km} \mathrm{~s}^{-1}$ with time. Such velocity shifts are observed if two maser lines forming a blend have intensity variations uncorrelated with each other. Alternatively, such velocity shifts might be explained by a single component, which changes its velocity due to ordered motion. However, we consider this as unlikely. In an expanding circumstellar shell the blueshifted components should show velocity shifts bluewards and the redshifted viceversa. The observed velocity shifts are however independent from the velocity of the components. Due to the large distances of several kpc (for estimates see: Herman \& Habing 1985a) of the stars we probably observe only the most luminous maser components. At a distance of $3 \mathrm{kpc}$, for example, the luminosity of an individual maser line has to surpass $10^{43}$ photons s$^{-1}$ to be detected during this monitor program. Weaker lines will have escaped detection. Thus, because of blending and limited sensitivity, the number of maser components in Tables 2 to 6 is a lower limit for the true number of components.

\subsubsection{Lifetimes of maser clouds}

Maser spectra observed within a few months usually show maser lines at the same velocities, but with different intensities. Intensity variations by a factor $2-5$ are common.
Maser spectra taken more than a year apart, may show an overall similar appearance, but the individual maser lines do not necessarily coincide in velocity anymore. The obvious interpretation of this observation is a limited lifetime for individual maser clouds of the order $\lesssim 1-3$ years. With outflow velocities of $\approx 7 \mathrm{~km} \mathrm{~s}^{-1}$ and $\approx 25 \mathrm{~km} \mathrm{~s}^{-1}$ for the two types of sources a lifetime $\approx 2$ years points to distances of $10^{13}$ to $10^{14} \mathrm{~cm}$ over which maser clouds may move, before they get destroyed. These distances are similar to their sizes, assuming that they are similar to the $\mathrm{H}_{2} \mathrm{O}$ maser clouds in other stars (Richards \& Yates 1998). This suggests that they do not survive in the outflow, but are continuously formed and destroyed.

Although individual maser lines seem to have a limited lifetime, new maser lines do not appear at random within the possible velocity range. Instead, they display a preference for showing up close to velocities at which maser lines were observed before. Regions in the circumstellar shell seem to maintain favorable conditions for maser emission (probably a favorable velocity structure) over longer times than typical lifetimes of maser components are. Therefore the presence of a few emission peaks in all our observations is not in contradiction with the proposed lifetimes. There is a fair chance that a maser line appears at almost the same velocity as a progenitor line, mimicking a longer lifetime. Although lifetimes of 10 years for particular lines cannot be ruled out, the case seems more convincing that the general lifetime of maser emitting clouds is closer to $1-3$ years than substantially longer. Strictly speaking, these lifetimes are given by the time intervals, during which the maser emitting clouds reach luminosities $\gtrsim 10^{43}$ photons $\mathrm{s}^{-1}$. They may exist for longer times at lower luminosity levels.

\subsubsection{Profile changes}

$\mathrm{OH} 15.7+0.8$ and $\mathrm{OH} 17.7-2.0$ did not show any preference for stronger maser intensities in the blue or red part of the velocity interval. Also the width of the interval seemed to be constant until the masers disappeared. For the other two sources the strongest masers were always in the blueshifted part of the velocity range. The only exception is the Feb. 99 spectrum of $\mathrm{OH} 12.8-0.9$ (Fig. 3). Although new maser components appeared during the monitoring program, their intensity remained comparable (within a factor of five) to earlier maser components at neighboring velocities. Thus, the overall spectral shapes have remained fairly constant since their discovery. For OH 12.8-0.9 we have significant and for $\mathrm{OH} 37.1-0.8$ we have marginally significant evidence that the $\mathrm{H}_{2} \mathrm{O}$ maser velocity range increases with time.

\section{Profiles and mass loss processes}

The $\mathrm{H}_{2} \mathrm{O}$ maser spectra of the four "non-variable OH/IR stars" are peculiar. On the AGB, where the stars possess an expanding radially symmetric circumstellar shell, the water molecules are located closer to the star than the $\mathrm{OH}$ 
molecules because $\mathrm{OH}$ is formed by destruction of $\mathrm{H}_{2} \mathrm{O}$ by the ambient interstellar UV-field in the outer part of the circumstellar shell (Huggins \& Glassgold 1982). As the expansion velocity increases radially outwards until it reaches a constant value the $\mathrm{H}_{2} \mathrm{O}$ maser velocity range has to fall inside the $\mathrm{OH}$ maser velocity interval. The masers of $\mathrm{OH} 12.8-0.9$ and $\mathrm{OH} 37.1-0.8$ are clearly at odds with the standard circumstellar shell model.

\subsection{Classification: young Proto-Planetary Nebulae}

The appearance of the $\mathrm{H}_{2} \mathrm{O}$ maser spectra changes in a definite way with increasing mass loss rates of the AGB stars. At low mass loss rates $\left(<10^{-6} M_{\odot} \mathrm{yr}^{-1}\right)$ the maser appears close to the radial velocity of the star (Type A spectra), while at higher mass loss rates the maser consists of two line complexes close to the velocities of the $\mathrm{OH}$ maser peaks (Type B spectra) (Engels et al. 1986; Takaba et al. 1994; Engels \& Lewis 1996). This evolution of the profile reflects the increase of the radius of the $\mathrm{H}_{2} \mathrm{O}$ maser shell with the improvement of dust shielding of the $\mathrm{H}_{2} \mathrm{O}$ molecules at high mass loss rates. At small radial distances the water vapor maser is excited in regions of large velocity gradient, forcing preferential propagation along tangential paths, whereas at larger radial distances the velocity gradient diminishes to the level that radial gain paths are preferred. A double-peaked $\mathrm{H}_{2} \mathrm{O}$ profile similar as for $\mathrm{OH}$ masers is shown therefore at high mass-loss rates. The appearance of Type A maser spectra in $\mathrm{OH} 17.7-2.0$ and $\mathrm{OH} 15.7+0.8$, two $\mathrm{OH} / \mathrm{IR}$ stars with large mass loss rates $\left(>10^{-5} M_{\odot} \mathrm{yr}^{-1}\right.$, according to their FIR energy distribution, Bedijn 1987), is therefore surprising.

The pecularity of the $\mathrm{H}_{2} \mathrm{O}$ maser spectra is not the first indication that these stars are not ordinary $\mathrm{OH} / \mathrm{IR}$ stars. Except for OH 12.8-0.9, all of them were found to be members of the class of "non-variable" OH/IR stars during the OH monitoring program of Herman \& Habing (1985b). These OH/IR stars are thought to have stopped their large amplitude variations and are developing now off the AGB with decreased mass loss rates (Bedijn 1987). The present observations reveal that the water vapor maser of $\mathrm{OH} 12.8-0.9$ has also not shown regular large amplitude variations, as it is common for ordinary OH/IR stars, so that all four stars can be considered as candidates for objects, which are in transition to the Planetary Nebula stage. OH 17.7-2.0 is the best observed object of our sample and may serve as a prototype for the class of "young PPNs". It has a classical OH maser spectrum and an overall spectral energy distribution (SED), which is bimodal. The inner part of the circumstellar dust shell has been diluted so far that the optical counterpart could be identified. The star has a spectral type K1-K4 with $T_{\text {eff }} \approx 4200 \mathrm{~K}$ (Le Bertre 1987). This effective temperature is much lower than those for the A-G stars present in most PPN candidates discussed in the literature. It suggests that the time elapsed since the end of the AGB evolution

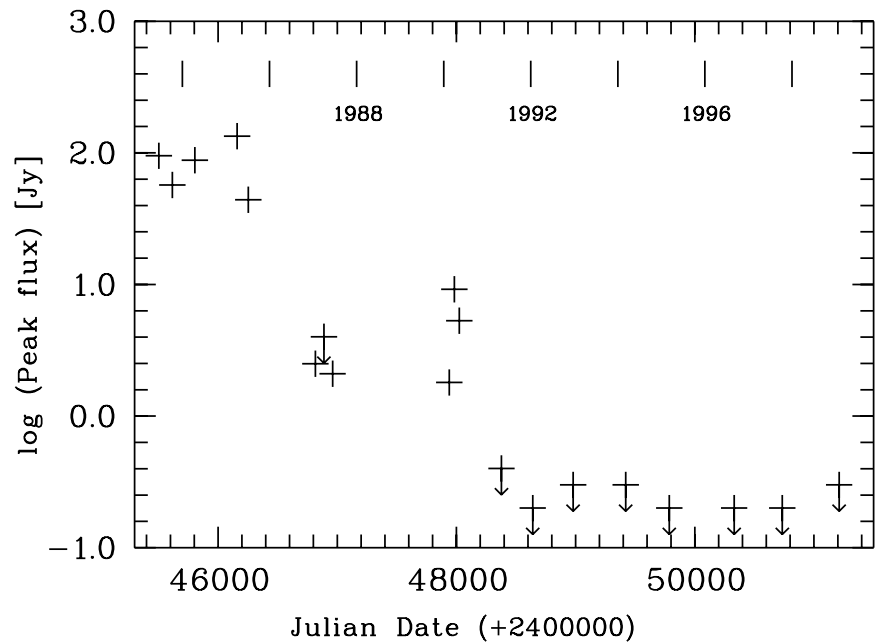

Fig. 5. $22 \mathrm{GHz} \mathrm{H}_{2} \mathrm{O}$ maser lightcurve of $\mathrm{OH} 17.7-2.0$ between 1983 and 1999. For each epoch the peak flux of the strongest line was taken. Data are from Nyman et al. (1986), Engels et al. (1986), Likkel (1989) and this work.

is shorter than for the hotter PPN candidates, qualifying them as "young PPNs".

\subsection{A dying $A G B$ wind}

Having qualified the evolutionary status of the stars as young PPNs, a straightforward interpretation of the $\mathrm{H}_{2} \mathrm{O}$ maser spectra is possible. The early Type A spectra of $\mathrm{OH} 17.7-2.0$ and $15.7+0.8$ are thought to originate in the old AGB wind, which had declined from the high massloss rate of $10^{-5} M_{\odot} \mathrm{yr}^{-1}$ at the end of the AGB to a value of $\approx 10^{-7} M_{\odot} \mathrm{yr}^{-1}$. The higher mass-loss rate is still witnessed by the FIR dust emission measured by IRAS and by the $\mathrm{OH}$ maser emission, while the lower mass-loss rate is an estimate based on the similarity of the $\mathrm{H}_{2} \mathrm{O}$ maser spectra of both stars with those of semiregular variable stars and blue Mira variables of this mass-loss rate.

In addition to the spectral shapes of the spectra, their time evolution is absolutely remarkable. From 1985 to 1990 the $\mathrm{H}_{2} \mathrm{O}$ maser of $\mathrm{OH} 17.7-2.0$ decreased in luminosity by a factor of hundred and is now undetectable since another six years (Fig. 5). From observations of semiregular and blue Mira variables we know that a threshold massloss rate somewhere between $10^{-8}$ and $10^{-7} M_{\odot} \mathrm{yr}^{-1}$ exists, below which $\mathrm{H}_{2} \mathrm{O}$ masers are not observed anymore (Szymczak \& Engels 1995, 1997; Benson \& Little-Marenin 1996). One is therefore tempted to attribute the decrease of $\mathrm{H}_{2} \mathrm{O}$ maser luminosity to a further rapid decrease of the mass loss rate in $\mathrm{OH} 17.7-2.0$ by a factor $\approx 10$ well below a level of $10^{-7} M_{\odot} \mathrm{yr}^{-1}$. The behaviour of the $\mathrm{H}_{2} \mathrm{O}$ maser of $\mathrm{OH} 15.7+0.8$ corroborates this result. Maybe maser lines will be detected again, but before they disappeared, $\mathrm{H}_{2} \mathrm{O}$ maser lines were detected always in both sources whenever they were observed. This supports the interpretation of the non-detections as being a systematic effect rather than a temporary variability fluctuation. 
The $\mathrm{H}_{2} \mathrm{O}$ maser spectra of both sources and their evolution give new evidence for a smooth transition of the mass-loss process at the end of the AGB evolution. The question, how the heavy mass loss at the tip of the AGB ends, was raised by Bedijn (1987) in the context of model fits to the optical-infrared SED of OH 17.7-2.0. He argued that some warm dust is needed close to the star and hence the mass loss cannot have stopped abruptly. A reasonable fit to the SED was obtained with an exponential decrease of $\dot{M}$ with a scale of 26.2 years and a drop of the mass loss rate from $10^{-3} M_{\odot} \mathrm{yr}^{-1}$ by a factor of $6 \times 10^{4}$ during the last 290 years. Van der Veen et al. (1989) followed this line of reasoning and found that the infrared SEDs of PPN candidates indicate post-AGB mass loss rates in the range $10^{-8}-10^{-5} M_{\odot} \mathrm{yr}^{-1}$ depending on the present day core mass of the star. These are achieved on time scales 600-1400 years after the start of the decline of the mass loss rate. Mass loss rates of the order $10^{-8}-10^{-7} M_{\odot} \mathrm{yr}^{-1}$ during the transition phase are also indicated by the $\mathrm{H} \alpha$ profiles in the spectra of Population II supergiant stars (Trams et al. 1989).

Evidence for rapid evolution of observable properties on timescales $\$ 100$ years as seen here in $\mathrm{OH} 17.7-2.0$ and probably $\mathrm{OH} 15.7+0.8$ has been observed also in $\mathrm{K} 3-35$, a very young PN (Miranda et al. 2001), and in the suspected PPN IRAS $18455+0448$ (Lewis et al. 2001). We conclude therefore that we have witnessed in $\mathrm{OH} 17.7-2.0$ and $\mathrm{OH} 15.7+0.8$ by accident the very short period of $\mathrm{H}_{2} \mathrm{O}$ maser emission from an AGB wind, dying out on the extremely short time scales proposed by Bedijn (1987). The OH masers of both stars were mapped in 1999 with MERLIN (Szymczak \& Richards 2001), showing that the conditions in the $\mathrm{OH}$ masing shell have not been altered since the discovery of the masers twenty years ago. The proposed rapid decline of the mass-loss rates witnessed by the $\mathrm{H}_{2} \mathrm{O}$ masers must therefore have started $\lesssim 10^{3}$ years ago, given the expansion velocities and $\mathrm{OH}$ shell sizes in these stars.

\subsection{A new axisymmetric Post-AGB wind}

$\mathrm{H}_{2} \mathrm{O}$ maser emission outside the $\mathrm{OH}$ maser velocity interval is incompatible with the standard radially symmetric circumstellar shell model. Axisymmetric winds (or bipolar jets) found in many PPNs and PNs suggest to interprete the high-velocity $\mathrm{H}_{2} \mathrm{O}$ masers of $\mathrm{OH} 12.8-0.9$ and OH 37.1-0.8 as early manifestations of the development of such a Post-AGB wind. Alternatively Gómez et al. (1994) discussed a model, in which the maser is located in a spherically symmetric Post-AGB wind close to the contracting central star. The velocity of the Post-AGB wind is increasing with time, so that the expansion velocity in the $\mathrm{H}_{2} \mathrm{O}$ maser zone is higher than in the $\mathrm{OH}$ maser zone. This is however a less likely explanation, because close to the star a large velocity gradient is expected and Type $\mathrm{A} \mathrm{H}_{2} \mathrm{O}$ maser spectra should be displayed. The masers of $\mathrm{OH} 12.8-0.9$ and $\mathrm{OH} 37.1-0.8$ are therefore interpreted as coming from a bipolar Post-AGB wind with $v_{\mathrm{e}} \approx 28 \mathrm{~km} \mathrm{~s}^{-1}$ (Table 1 ), where $v_{\mathrm{e}}$ is the (projected) wind velocity. While the masers of $\mathrm{OH} 15.7+0.8$ and $\mathrm{OH} 17.7-2.0$ are signposts of the old "dying AGB wind", these two masers are proposed to emerge from the new Post-AGB wind.

In principle, the Type A and the high-velocity masers could be unified in one model, assuming that the former are generated in the Post-AGB wind with a bipolar flow as well, but with the flow axis orientated almost perpendicular to the line of sight. No large velocity dispersion would be observed then. Such a configuration has been observed in K3-35 by Miranda et al. (2001). However, the completely different development with time of the Type A masers argues against it.

\subsubsection{Cousins of "water fountain" sources?}

The high-velocity masers show strong similarity to the "water fountain" sources IRAS 15405-4945, 16342-3814, 19134+2131, and W43A (te Lintel Hekkert et al. 1988; Likkel \& Morris 1988). These sources display water vapor masers and in part $\mathrm{OH}$ masers with velocity ranges of 130 $260 \mathrm{~km} \mathrm{~s}^{-1}$. One is tempted to classify $\mathrm{OH} 12.8-0.9$ and OH 37.1-0.8 as earlier evolutionary stages of this class of sources. Their $\mathrm{H}_{2} \mathrm{O}$ maser velocities may increase further with growing velocities of the Post-AGB wind, the $\mathrm{OH}$ maser of the remnant AGB wind may eventually disappear and high-velocity $\mathrm{OH}$ masers possibly might be excited in the future. Monitoring of $\mathrm{OH} 12.8-0.9$ and $\mathrm{OH} 37.1-0.8$ will be needed to confirm the evidence gathered already for an increase of the $\mathrm{H}_{2} \mathrm{O}$ maser velocity range with time (cf. Sect. 4.5.3).

Likkel et al. (1992) have monitored the $\mathrm{H}_{2} \mathrm{O}$ masers of three of the four "water fountain" sources over two years. Their analysis of the spectra yielded very similar results compared with those obtained in Sect. 4. They found that the individual lines varied in intensity at fixed velocities, and that they may have disappeared at some velocities, while other lines appeared or reappeared. Apparent velocity shifts were attributed to intensity variations of two or more lines closely spaced in velocity instead to a change in velocity of an individual component. Some lines have retained their identity over the two years of the monitoring program, showing that also the lifetimes of individual maser lines are compatible with the 1-3 years, which were derived for the sources of the present paper. Summarizing, the spectral shape and their variations with time are very similar to the high velocity masers discussed here.

\section{Discussion: Masers and PPNs}

\subsection{Masers and search strategies for PPNs}

Two methods using IRAS data have been employed to find PPN candidates so far. The first searched for optically visible stars with large infrared excesses, the second searched in particular areas of the IRAS color-color 


\section{IRAS Color-Color Plot}

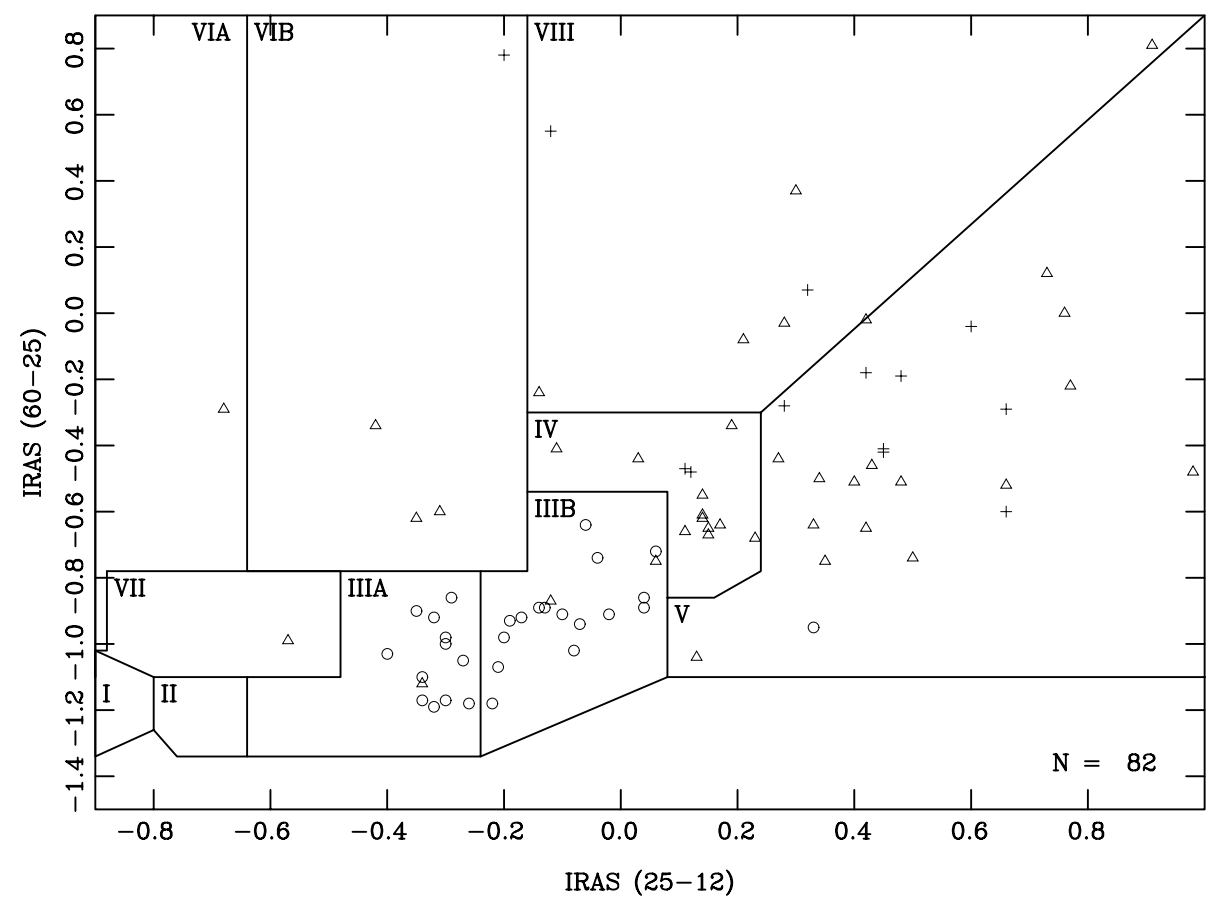

Fig. 6. PPN (candidates) in the IRAS color-color diagram. (+) "non-variable" OH/IR stars from Herman \& Habing (1985b); (o) suspected PPNs from the Arecibo sample of OH/IR stars (Lewis 1989; Engels \& Lewis 1996); ( $\Delta$ ) PPNs from Nyman et al. (1998).

diagrams for very cool infrared sources (cf. Kwok 1993 and references therein). Several studies have shown that with a decrease of the high mass loss rates at the tip of the AGB the stars evolve toward redder colors (Bedijn 1987; Volk \& Kwok 1989). Hence, PPNs are thought to populate mainly areas IV and V in the IRAS(60-25) vs. (25-12) plot (cf. Fig. 6) redwards of region III, in which most of the variable $\mathrm{OH} / \mathrm{IR}$ stars are located. Herman \& Habing (1985b) monitored the $1612 \mathrm{MHz}$ OH maser emission of a sample of $\mathrm{OH} / \mathrm{IR}$ stars and found that almost all of the non-variable objects are located in the regions thought to be populated by PPNs (Fig. 6). This observation gave cause to the view, that the cessation of large amplitude variability and the end of the phase of high mass loss rates ("superwind phase") are closely linked.

However, PPN candidates are not restricted to these areas (Volk 1992; van Hoof et al. 1997) and it is difficult to separate them by their IRAS colors elsewhere in the colorcolor plot. In the PPN compilation of Nyman et al. (1998) for example, quite a number of sources are not in the very red part of Fig. 6. The same is found by Szczerba et al. (2001) for a recent compilation of about 220 PPN candidates. In the IIIb area, where heavily reddened $\mathrm{OH} / \mathrm{IR}$ stars prevail, candidates for very young PPNs may hide and will not be identifiable unless observations to prove the cessation of the large amplitude variability are made.

Under these circumstances searches for peculiar maser spectra may help. In the case of OH masers Lewis (1989) has presented a list of PPN candidates from the Arecibo sample of $\mathrm{OH} / \mathrm{IR}$ stars in region III based mainly on the presence of main-line maser emission being stronger than the usually dominating $1612 \mathrm{MHz}$ satellite emission (Fig. 6). Zijlstra et al. (2001) compiled a list of sources having $\mathrm{OH}$ spectra with a wide velocity range $\left(\Delta v^{\mathrm{OH}} \geq 50 \mathrm{~km} \mathrm{~s}^{-1}\right)$ and profiles deviating from the standard double-peaked appearance. Objects from this list studied interferometrically show evidence for bipolar outflows.

The search for peculiar $\mathrm{H}_{2} \mathrm{O}$ maser spectra would be another way to identify very young PPN candidates. Engels \& Lewis (1996) identified 7 out of $382 \mathrm{OH} / \mathrm{IR}$ stars of the Arecibo sample from region III to have Type A maser spectra in stars where Type B spectra are expected (Fig. 6) Two of them appear as PPN candidates otherwise in the literature. Engels (1996) argued that IRAS $19296+2227$ is a PPN based on the properties of its $\mathrm{H}_{2} \mathrm{O}$ maser emission. $19296+2227$ is located in region III, has a peculiar $1612 \mathrm{MHz} \mathrm{OH}$ profile, and has a Type $\mathrm{A}_{2} \mathrm{O}$ maser.

$\mathrm{SiO}$ maser emission is rarely detected among PPN candidates (Nyman et al. 1998). Secure detections were made only in $\mathrm{OH} 231.8+4.2$ at $43 \mathrm{GHz}$ by Jewell et al. (1991) and in IRAS $19312+1950$ at 43 and $86 \mathrm{GHz}$ by Nakashima \& Deguchi (2000). In accordance with its PPN classifica-

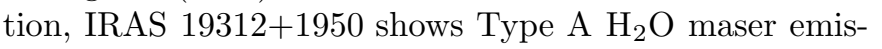
sion. Because of its rareness $\mathrm{SiO}$ maser emission is unsuitable to search for PPNs.

Although only a minority of stars actually leaving the AGB may display masers, these might be discovered easily because their peculiar profiles will tell. 


\subsection{The onset of PPN evolution}

The termination of the AGB evolution and the onset of the PPN phase is difficult to study observationally because the star is hidden optically during this transition. It is also not clear which stars do exhibit this transition currently and how reliable the PPN classifications of many objects are. Accordingly the assignment of individual stars to evolutionary stages either at the end of the AGB or at the beginning of the PPN phase is fuzzy and varies in the literature. Volk \& Kwok (1989) assume that large-scale mass loss will not be possible after a decrease of the stellar envelope mass below $10^{-3} M_{\odot}$. They define this point, at which the star already has a temperature of $T_{\text {eff }} \approx 5000 \mathrm{~K}$, as end of the AGB and the beginning of the PPN phase. Kwok (1993) assumed that the star is observationally visible already at this transition point and developed criteria to characterize PPNs, which emphasize the reappearance of the optical emission as a consequence of the dilution of the circumstellar shell.

In early models of the transition process the switch from the late AGB wind ("superwind") to a new wind with decreased mass loss rates was made instantaneously (Volk \& Kwok 1989; Vassiliadis \& Wood 1994). Blöcker (1995) was the first, who included the step down of the mass loss rate over a few to several hundred years (depending on the model) in his calculations, and found improvements in the modeling of the infrared energy distributions of PPNs. It is the end of the mass loss reduction phase, which is considered in his models as the end of the AGB evolution. The Blöcker model for a $3 M_{\odot}$ star predicts a transition duration from a totally obscured $\mathrm{OH} / \mathrm{IR}$ star to a double-peaked energy distribution expected for PPNs of $\approx 500$ years (Steffen et al. 1998).

The "non-variable" OH/IR stars then are to be considered as stars in the mass loss reduction phase, e.g. as stars currently departing from the AGB. OH $15.7+0.8$ and 37.1-0.8 were already considered as misclassified $\mathrm{OH} / \mathrm{IR}$ stars by Lewis \& te Lintel Hekkert (1991), being PPN candidates instead. Also Hu et al. (1993) prefer the end of the large amplitude variability and the associated drop of the mass-loss rates as definition for the end of the AGB and the beginning of the PPN phase. This definition has been adopted here referring to the four objects discussed as "young PPNs". In IRAS 16342-3814, one of the "water fountain" sources, the near-infrared counterpart was found only recently with HST (Sahai et al. 1999), and it was shown that the star is hidden by a torus-like distribution of circumstellar dust. It is therefore probable that the visibility of the central star depends not merely on evolutionary stage but also on the inclination of the hiding dust geometry with respect to the line of sight. A simple relation between the observed SED and the evolutionary stage, as it comes out from spherical symmetric models, might not be given. The cessation of the high-amplitude pulsations is therefore a less ambiguous criterium to classify PPNs.
The "young PPNs" phase is considered to be short $\left(<10^{3}\right.$ years $)$, as the regions in which the $\mathrm{OH}$ maser emission emerges, are still undisturbed. It is therefore surprising that in the sample of $\mathrm{OH} / \mathrm{IR}$ stars monitored by Herman \& Habing (1985b) almost half classify as "nonvariable" or as variable with small amplitude, implying a similar duration for the "pulsating" phase on the AGB. This might be explained by strong selection effects of the monitored sample toward high main-sequence mass progenitors $\left(\approx 3-8 M_{\odot}\right)$ and a "pulsating" phase connected with a "superwind" $\left(\dot{M}>10^{-5} M_{\odot} \mathrm{yr}^{-1}\right)$ of a few hundred years. The monitored sample was drawn from a blind survey along the galactic plane for $1612 \mathrm{MHz} \mathrm{OH}$ maser emission by Baud et al. (1979) with a flux limit of $\approx 2$ Jy. The kinematic distances derived for the newly discovered $\mathrm{OH} / \mathrm{IR}$ stars were several kiloparsec, inferring rather high bolometric luminosities. There were no systematic differences in distance distribution with respect to the variability properties. Combined with their closeness to the galactic plane these stars probably descend from stars with main-sequence masses higher than classical Mira variables. A prototype is $\mathrm{OH} 26.5+0.6$ with a period of 1600 days, for which Justtanont et al. (1996) found that the currently experienced "superwind" emerged only less than 150 years ago. The equipartion of "variable" and "non-variable" OH/IR stars in the sample of Herman \& Habing might therefore be due to sampling only the latest stages of the most massive stars, which develop through the AGB.

An alternative explanation for the relatively large number of "non-variable" $\mathrm{OH} / \mathrm{IR}$ stars might be a recurrent development through a non-pulsating phase after the occurrence of thermal pulses. High-amplitude pulsation and high mass loss rates are thought to stop after a pulse and the star might mimic for a while a star leaving the AGB after the last pulse. Calculations of the varying SED during a pulse cycle failed however to generate the very red colors in the IRAS color-color diagram, where the "non-variable" OH/IR stars were found (Steffen et al. 1998).

\subsection{Bipolar outflows}

The apparently coeval support of two winds in an evolutionary phase with a lifetime $\$ 1000$ years is astonishing. It suggests that within this time range the AGB wind dies out, while a new Post-AGB wind emerges. According to the Interacting Stellar Wind (ISW) model (cf. Frank 1999) the Post-AGB wind will acquire a bipolar shape, if the AGB wind has an aspherical axi-symmetric density distribution. The flow is shaped by the interaction of the two winds, traveling faster into the more tenuous regions of the AGB wind. A variation of this model postulates the presence of a mechanism, possibly connected to magnetic fields, which could launch and collimate the Post-AGB outflow (Blackman et al. 2001). 
Evidence for asphericity of the AGB wind forming circumstellar shells is increasing, but it is unknown, when it develops and if the implied density gradient is large enough. Josselin et al. (2000) found strong asymmetries in the gas distribution of Mira and concludes that asymmetrical mass loss processes occur already early in the AGB evolution. On the other hand circumstellar arcs discovered around PPNs suggest that the wind of the progenitor AGB stars was spherical symmetric, and that the transition to a bipolar morphology has occurred only during a short period at the end of the AGB evolution (Hrivnak et al. 2001).

These contradicting observations might be explained by the presence of other factors playing a significant role for the details of the mass loss process transition. Mastrodemos \& Morris (1999) have shown that the presence of a detached binary companion has a significant influence on the morphology of the circumstellar shell created by a spherical symmetric AGB wind. The presence of a binary companion naturally explains the formation of an axi-symmetric density distribution required for the ISW mechanism to work.

Kastner et al. (1996) found observationally that bipolar outflows are more likely associated with larger progenitor masses, and PN formation models from Mellema (1997) using evolutionary tracks with different central star masses confirm this correlation. Thus, the different maser types observed may not be succeeding phases of the transition process but rather manifestations of the transition process in stars with or without binary companion and/or with different central star masses. The possibly bipolar maser outflows as in $\mathrm{OH} 12.8-0.9$ and $\mathrm{OH} 37.1-0.8$ might then indicate higher central star masses compared to the other two sources or they may differ because of the presence of a binary companion.

To circumvent problems of the ISW model in explaining observations of point-symmetric PN morphologies Sahai \& Trauger (1998) proposed the presence of highspeed (few $100 \mathrm{~km} \mathrm{~s}^{-1}$ ) collimated outflows, which carve out imprints within an intrinsic spherical AGB circumstellar shell. These outflows are thought to operate during the late-AGB or young PPN evolutionary phase and define the directions into which later the hot and even faster PN wind will expand. Observational evidence for such collimated outflows on the AGB are lacking so far, but if real, objects like $\mathrm{OH} 12.8-0.9$ and $\mathrm{OH} 37.1-0.8$ and other $\mathrm{OH} / \mathrm{IR}$ stars with bipolar outflows (Zijlstra et al. 2001) might belong to the earliest transition phases, in which these collimated outflows are observed.

\subsection{Other masers - chronological sequence}

The end of the large amplitude pulsation is accompanied by a change of the maser properties, which is probably caused by the changes, which the mass loss process is undergoing in response to the declining pulsation of the central star. Lewis (1989) already noted that the main line $\mathrm{OH}$ maser emission is often stronger in PPNs than in $\mathrm{OH} / \mathrm{IR}$ stars. In his "chronological sequence" of maser properties he considers the reappearance of stronger main line emission among very red IRAS sources as a strong indication for a PPN nature. Using the presence and absence of particular masers $\left(\mathrm{SiO}, \mathrm{H}_{2} \mathrm{O}, \mathrm{OH}\right)$ as a clock to determine the evolutionary stage of the star on the AGB and beyond is an appealing concept, but will fail as soon as short timescales get involved and the spherical symmetry is lost. On the AGB with increasing mass loss rates $\mathrm{SiO}$, $\mathrm{H}_{2} \mathrm{O}$, main line $\mathrm{OH}$ and finally satellite line $\mathrm{OH}$ masers are added. Beyond the end of the AGB a reversion of the "chronological sequence" is expected in a way that main line $\mathrm{OH}, \mathrm{H}_{2} \mathrm{O}$, and $\mathrm{SiO}$ masers reappear with decreasing mass loss rates. The short timescales involved compared to the dynamical timescales in the circumstellar shell may lead to a gradient of mass loss rates across the shell, destroying any systematics in the appearance of masers. For example, as the $\mathrm{H}_{2} \mathrm{O}$ masers of $\mathrm{OH} 15.7+0.8$ and $\mathrm{OH} 17.7-$ 2.0 are considered here to be evidence for low mass loss rates, also the main line $\mathrm{OH}$ masers are expected to become stronger than the satellite lines, as it is often the case for Mira variables. As of 1987 however this has not happened (Dickinson \& Turner 1991).

The occurrence of high-velocity masers associated with bipolar outflows (Zijlstra et al. 2001; also OH 12.8-0.9, OH 37.1-0.8, and the "water fountain" sources) indicates a break of spherical symmetry at the end of the AGB and the inclination of the new (bipolar) outflows to the line of sight might well be a significant factor governing the observability of masers. A survey of maser properties of PPNs (Engels 2001) failed probably therefore to establish firm correlations with particular stages of the transition process.

\section{Conclusions}

Four "non-variable OH/IR stars" were monitored over more than 10 years in the $22 \mathrm{GHz}$ water vapor maser line and all four objects show maser properties deviant from ordinary variable $\mathrm{OH} / \mathrm{IR}$ stars. In $\mathrm{OH} 15.7+0.8$ and $\mathrm{OH} 17.7-2.0$ the maser flux decreased and finally disappeared. Their maser probably originated from the fading AGB wind at the very end of the AGB evolution. In $\mathrm{OH} 12.8-0.9$ and $\mathrm{OH} 37.1-0.8$ the masers probably trace a new faster (bipolar) Post-AGB wind. All four objects are therefore currently leaving the Asymptotic Giant Branch and are entering the Proto-Planetary Nebula phase. Given the appearance of the yet undisturbed $1612 \mathrm{MHz} \mathrm{OH}$ masers the decline of the mass-loss rates and the emergence of the new wind occurred less than $\approx 1000$ years ago.

The majority of known maser sources associated with the AGB, in particular of $\mathrm{OH}$, were discovered by surveys conducted more than twenty years ago. The case of the $\mathrm{H}_{2} \mathrm{O}$ maser of $\mathrm{OH} 17.7-2.0$ gives evidence for the disappearance of a once strong maser on the time scale of years. Another case exhibiting a rapid maser flux decline, this time in OH, is IRAS $18455+0448$, which is also 
interpreted as result of the current decline of the massloss rate initiating the departure from the AGB (Lewis et al. 2001). In both sources the decline was discovered by accident during non-systematic revisits. Redoing maser surveys on timescales of decades might be rewarding by uncovering sources, in which fast evolutionary processes currently are going on. Such surveys need to cover also objects not detected as masers before, to find also objects, in which masers turn on. As many of them might be optically invisible, mapping of the masers will be an important tool to improve our understanding of the mass loss geometry in objects currently departing from the AGB.

Acknowledgements. This research has made use of the Simbad database, operated at CDS, Strasbourg, France. I thank R. Cesaroni, B. M. Lewis and K. Schönberg for help with the observations in Effelsberg. The careful assessment of the paper by the referee H. Habing is acknowledged.

\section{References}

Baud, B., Habing, H. J., Matthews, H. E., \& Winnberg, A. 1979, A\&AS, 36, 193

Bedijn, P. J. 1987, A\&A, 186, 136

Benson, P. J., \& Little-Marenin, I. R. 1996, ApJS, 106, 579

Blackman, E. G., Frank, A., Markiel, J. A., Thomas, J. H., \& van Horn, H. M. 2001, Nature, 409, 485

Blöcker, T. 1995, A\&A, 299, 755

Bowers, P. F., Reid, M. J., Johnston, K. J., Spencer, J. H., \& Moran, J. M. 1980, ApJ, 242, 1088; (Erratum: ApJ, 248, 880)

Bowers, P. F., Johnston, K. J., \& Spencer, J. H. 1981, Nature, 291, 382

Bowers, P. F., \& de Jong, T. 1983, AJ, 88, 655

Cato, B. T., Rönnäng, B. O., Rydbeck, O. E. H., et al. 1976, ApJ, 208, 87

Dickinson, D. F., \& Turner, B. E. 1991, ApJS, 75, 1323

Engels, D., Schmid-Burgk, J., \& Walmsley, C. M. 1986, A\&A, 167, 129

Engels, D., \& Lewis, B. M. 1996, A\&AS, 116, 117

Engels, D. 1996, A\&A, 315, 521

Engels, D. 1997, in Planetary Nebulae, ed. H. J., Habing, \& H. J. G. L. M. Lamers (Kluwer), IAU Symp., 180, 348

Engels, D. 2001, in Post-AGB objects as a phase of stellar evolution, ed. R. Szczerba, \& S. K. Górny (Kluwer), 417

Fix, J. D., \& Mutel, R. L. 1984, AJ, 89, 406

Frank, A. 1999, New Astron. Rev., 43, 31

Gómez, Y., Rodriguez, L. F., Contréras, M. E., \& Moran, J. M. 1994, Rev. Mex. Astron. Astroph., 28, 97

Habing, H. J., van der Veen, W., \& Geballe, T. 1987, in Late Stages of Stellar Evolution, ed. S. Kwok, \& S. R. Pottasch (Reidel), 91

Herman, J., \& Habing, H. J. 1985a, Phys. Rep., 125, 255

Herman, J., \& Habing, H. J. 1985b, A\&AS, 59, 523

Herman, J., Baud, B., Habing, H. J., \& Winnberg, A. 1985, A\&A, 143, 122

Heske, A., Forveille, T., Omont, A., van der Veen, W. E. C. J., \& Habing, H. J. 1990, A\&A, 239, 173

Hrivnak, B. J., Kwok, S., \& Su, K. Y. L. 2001, AJ, 121, 2775

Hu, J. Y., Slijkhuis, S., de Jong, T., \& Jiang, B. W. 1993, A\&AS, 100, 413
Huggins, P. J., \& Glassgold, A. E. 1982, AJ, 87, 1828

Jewell, P. R., Snyder, L. E., Walmsley, C. M., Wilson, T. L., \& Gensheimer, P. D. 1991, A\&A, 242, 211

Josselin, E., Mauron, N., Planesas, P., \& Bachiller, R. 2000, A\&A, 362, 255

Justtanont, K., Skinner, C. J., Tielens, A. G. G. M., Meixner, M., \& Baas, F. 1996, ApJ, 456, 337

Kastner, J. H., Weintraub, D. A., Gatley, I., Merrill, K. M., \& Probst, R. G. 1996, ApJ, 462, 777

Kwok, S. 1993, ARA\&A, 31, 63

Le Bertre, T. 1987, A\&A, 180, 160

Lewis, B. M. 1989, ApJ, 338, 234

Lewis, B. M., \& te Lintel Hekkert, P. 1991, Proc. Astron. Soc. Aust., 9, 304

Lewis, B. M. 1994, ApJS, 93, 549

Lewis, B. M., Oppenheimer, B. D., \& Daubar, I. J. 2001, ApJ, 548, L77

Likkel, L., \& Morris, M. 1988, ApJ, 329, 914

Likkel, L. 1989, ApJ, 344, 350

Likkel, L., Morris, M., \& Maddalena, R. J. 1992, A\&A, 256, 581

Mastrodemos, N., \& Morris, M. 1999, ApJ, 523, 357

Mellema, G. 1997, A\&A, 321, L29

Miranda, L. F., Gómez, Y., Anglada, G., \& Torrelles, J. M. 2001, Nature, 414, 284

Nakashima, J., \& Deguchi, S. 2000, PASJ, 52, L43

Nyman, L-Å., Johansson, L. E. B., \& Booth, R. S. 1986, A\&A, 160,352

Nyman, L-Å., Hall, P. J., \& Olofsson, H. 1998, A\&AS, 127, 185

Richards, A. M. S., \& Yates, J. A. 1998, Ir. Astr. J., 25, 7

Sahai, R., \& Trauger, J. T. 1998, AJ, 116, 1357

Sahai, R., te Lintel Hekkert, P., Morris, M., et al. 1999, ApJ, 514, L115

Steffen, M., Szczerba, R., \& Schönberner, D. 1998, A\&A, 337, 149

Szczerba, R., Górny, S. K, \& Zalfresso-Jundzillo, M. 2001, in Post-AGB objects as a phase of stellar evolution, ed. R. Szczerba, \& S. K. Górny (Kluwer), 13

Szymczak, M., \& Engels, D. 1995, A\&A, 296, 727

Szymczak, M., \& Engels, D. 1997, A\&A, 322, 159

Szymczak, M., \& Richards, A. M. S. 2001, in Post-AGB objects as a phase of stellar evolution, ed. R. Szczerba, \& S. K. Górny (Kluwer), 429

Takaba, H., Ukita, N., Miyaji, T., \& Miyoshi, M. 1994, PASJ, 46,629

te Lintel Hekkert, P., Habing, H. J., Caswell, J. L., Norris, R. P., \& Haynes, R. F. 1988, A\&A, 202, L19

te Lintel Hekkert, P., Chapman, J. M., \& Zijlstra, A. A. 1992, ApJ, 390, L23

Trams, N. R., Waters, L. B. F. M., Waelkens, C., Lamers, H. J. G. L. M., \& van der Veen, W. E. C. J. 1989, A\&A, 218, L1

van der Veen, W. E. C. J., Habing, H. J., \& Geballe, T. R. 1989, A\&A, 226, 108

van Hoof, P. A. M., Oudmaijer, R. D., \& Waters, L. B. F. M. 1997, MNRAS, 289, 371

Vassiliadis, E., \& Wood, P. R. 1994, ApJS, 92, 125

Volk, K. M., \& Kwok, S. 1989, ApJ, 342, 345

Volk, K. 1992, ApJS, 80, 347

Zijlstra, A. A., Chapman, J. M., te Lintel Hekkert, P., et al. 2001, MNRAS, 322, 280 